\title{
State Dependence of Spike Timing and Neuronal Function in a Motor Pattern Generating Network
}

\author{
Jin-sheng Wu, Michael R. Due, Kosei Sasaki, Alex Proekt, Jian Jing, and Klaudiusz R. Weiss \\ Department of Neuroscience, Mount Sinai School of Medicine, New York, New York 10029
}

When sustained firing of a neuron is similar in different types of motor programs, its role in the generation of these programs is often similar. We investigated whether this is also the case for neurons involved in phase transition. In the Aplysia feeding central pattern generator (CPG), identified interneuron B64 starts firing at the transition between the protraction and the retraction phases of all types of motor programs, and its firing is sustained during the retraction phase. It was thought that B64 functions as a protraction terminator as it provides strong inhibitory input to protraction interneurons and motoneurons. Furthermore, premature activation of B64 can lead to premature termination of the protraction phase. Indeed, as we show here, B64 can terminate the protraction phase regardless of the type of motor program. However, B64 actually only functions as a protraction terminator in ingestive-like but not in egestive-like programs. This differential role of B64 results from a differential timing of the initiation of B64 spiking in the two types of programs. In turn, this differential timing of the initiation of B64 firing is determined by the internal state of the CPG. Thus, this study indicates the importance of the timing of initiation of firing in determining the functional role of a neuron and demonstrates that this role depends on the activity-dependent state of the network.

Key words: Aplysia; central pattern generator; feeding; network state; phase transition; motor programs

\section{Introduction}

Depending on the inputs or internal states, many motor networks or central pattern generators (CPGs) produce different types of motor programs (Getting, 1989; Marder and Calabrese, 1996; Nusbaum and Beenhakker, 2002; Grillner, 2006). Distinct programs can be generated by altering the activity of a subset of CPG elements whose sustained firing is either differentially recruited into or switched between specific phases in different motor programs (Flamm and Harris-Warrick, 1986; Nusbaum and Marder, 1989; Berkowitz and Stein, 1994; Combes et al., 1999; Jing and Weiss, 2002, 2005; Pena et al., 2004). However, to function differently, principal CPG neurons need not show a major change of phasing of sustained firing (Saideman et al., 2006; Thirumalai et al., 2006). Of particular interests are phase-transition neurons, i.e., neurons that terminate one phase of the program and initiate the next phase. Conceivably, the same neuron within this group of neurons could function as a phase terminator when its activity began before, but not after, phase transition occurred. Such a possibility is difficult to test, especially in vertebrate networks, in which a large number of neurons may be active at the time when phase transitions occur. Here, we exploited the experimentally advantageous features of the Aplysia feeding network to determine whether the timing of initiation of firing of an identi-

Received March 12, 2007; revised Aug. 26, 2007; accepted Aug. 27, 2007.

This work was supported by the National Institutes of Health Grant MH 36730. We thank Dr. Elizabeth C. Cropper for comments on a previous version of this manuscript.

Correspondence should be addressed to Dr. Klaudiusz R. Weiss, Department of Neuroscience, Box 1065, Mt. Sinai School of Medicine, 1 Gustave Levy Place, New York, NY 10029. E-mail: klaudiusz.weiss@mssm.edu.

D0I:10.1523/JNEUROSCI.1806-07.2007

Copyright $\odot 2007$ Society for Neuroscience ～0270-6474/07/2710818-14\$15.00/0 fied phase-transition neuron may differ when the network is activated by different stimuli or is in a different activity-dependent state, and whether this difference in timing may alter the functional role that this neuron plays in generating motor programs.

Specifically, we investigate how network states and the timing of the initiation of firing may influence the function of interneuron $\mathrm{B} 64$ during the transition from the protraction to the retraction phase in ingestive and egestive feeding motor programs. Because the bulk of sustained firing of B64 occurs during the retraction phase in all types of motor programs and B64 firing can terminate the protraction phase, the timing of the onset of B64 firing could determine whether B64 acts, or does not act, as a protraction terminator in different programs. Using a series of experimental manipulations, we find that B64 actually acts as a protraction-phase terminator in ingestive-like but not in egestive-like programs. Notably, in ingestive programs, B64 fires before protraction termination, whereas in egestive programs, B64 fires after protraction termination. Furthermore, the timing of the onset of B64 firing depends on the activity-dependent state of the CPG rather than the input used to elicit the response. When peripheral structures are present, sensory afferents have been shown to influence motor patterns by initiating phase transition (Pearson, 1995; Rossignol et al., 2006), presumably by influencing the spike timing of relevant CPG elements. However, the effects of activity-dependent network states on the timing of activation of phase-transition CPG elements have not yet been demonstrated. Thus, our study extends the range of statedependent actions that are used to influence the output of the feeding CPG and indicates the importance of spike-timing processes in determining the functional role that individual neurons play in different programs. 

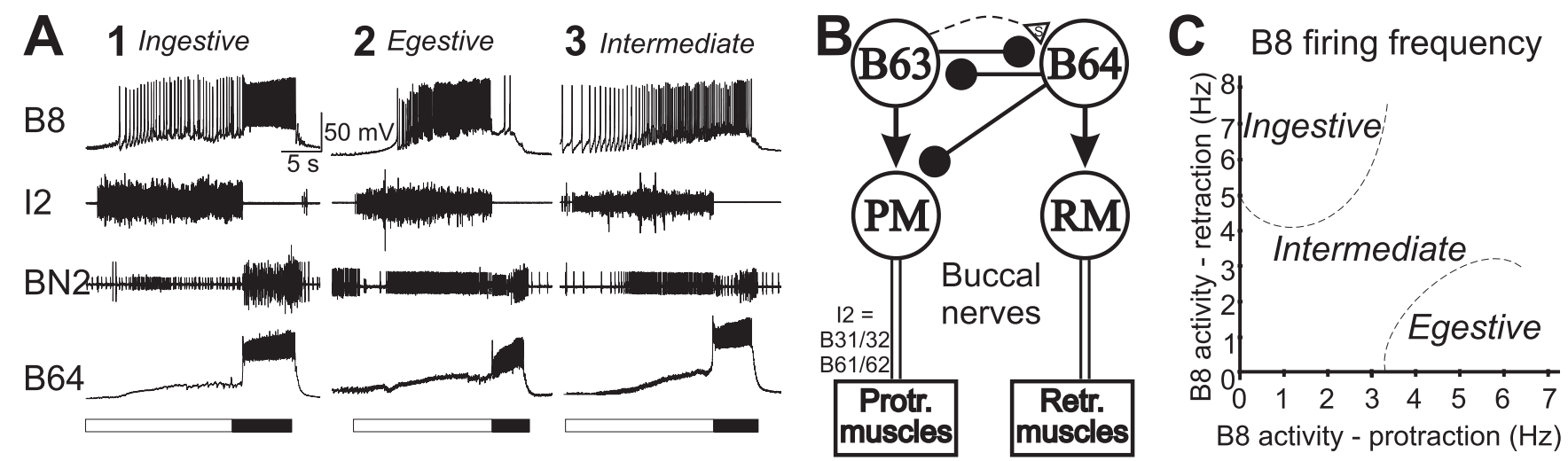

Figure 1. Classification of feeding motor programs and B64 activity in different types of programs. $A$, Examples of three types of motor programs elicited by $(B \mid-2$ stimulation: ingestive, egestive, and intermediate. Protraction (open bar) was monitored by activity in the 2 nerve. Retraction (filled bar) was monitored by activity in BN2 after protraction. B64 displayed robust activity throughout the retraction phase in all three types of motor programs and therefore has been used as a monitor for retraction in previous studies. In the ingestive program (A1), the radula closer motoneuron $B 8$ fired predominantly during the retraction phase; in the egestive program (A2), B8 fired vigorously during the protraction phase; in the intermediate program (A3), B8 was active during both protraction and retraction. $B$, Schematic diagrams of the core circuit (protraction-retraction) mediating feeding motor programs. B63 interneuron is active during protraction in all types of motor programs, inhibits the B64 interneuron, and drives activity in protraction motoneurons (PM) B31/32 and B61/62, which in turn innervate protraction muscles (Protr. muscles). B64 is active during retraction, inhibits protraction interneuron B63 and protraction motoneurons, and provides excitation to retraction motoneurons (RM), which in turn innervate retraction muscles (Retr. muscles). Phase transition from protraction to retraction is mediated, at least in part, by slow excitation of B64 by B63, which, with a delay, overcomes fast inhibition and eventually triggers B64 spiking that in turn inhibits protraction interneurons and motoneurons to terminate protraction and initiate retraction. Connections: open triangle, excitation; filled circle, inhibition; 5 , slow synaptic connections; arrows, excitatory pathways that may include both chemical and electrical connections; broken line, polysynaptic connection. C, Plot of B8 activity during protraction versus during retraction illustrating cluster boundaries for ingestive and egestive motor programs. Intermediate programs are motor programs that are between clusters for ingestive and egestive programs.

\section{Materials and Methods}

Experiments were performed on Aplysia californica obtained from Marinus (Long Beach, CA). Aplysia were maintained in holding tanks circulating artificial seawater (ASW) made from Instant Ocean (Aquarium Systems, Mentor, $\mathrm{OH}$ ) at $14-15^{\circ} \mathrm{C}$. Animals weighing $100-250 \mathrm{~g}$ were anesthetized by injection of isotonic $\mathrm{MgCl}_{2}$ solution ( $50 \%$ of body weight) into the body cavity. The cerebral ganglia and buccal ganglia were dissected out with the cerebral-to-buccal connectives intact. The ganglia were pinned to a silicone elastomer (Sylgard; Dow Corning, Midland, MI) that bottom lined a dish filled with a dissection solution consisting of $50 \%$ ASW (in mM: $460 \mathrm{NaCl}, 10 \mathrm{KCl}, 55 \mathrm{MgCl}_{2}, 11 \mathrm{CaCl}_{2}$, and 10 HEPES buffer, $\mathrm{pH}$ 7.6) and $50 \%$ isotonic $\mathrm{MgCl}_{2}$ solution at room temperature. Cerebral ganglia were pinned ventral surface up, and buccal ganglia were pinned rostral surface up. The connective tissue sheath that covers the neurons was surgically removed from both ganglia from which the recordings were to be made, and the dissection solution was then replaced with 100\% ASW. The ganglia were then transferred to the recording chamber containing $\sim 1.5 \mathrm{ml}$ of ASW. During all experiments, the ganglia were maintained at $14-16^{\circ} \mathrm{C}$ and continuously perfused with ASW at the rate of $\sim 0.3 \mathrm{ml} / \mathrm{min}$ using a peristaltic pump. Excess solution was removed by vacuum suction. All chemicals were purchased from Sigma (St. Louis, MO).

Intracellular recordings were performed using single-barreled microelectrodes filled with $2 \mathrm{M}$ potassium acetate and beveled to a resistance of 5-8 M $\Omega$, using either an Axoclamp 2A (Molecular Devices, Sunnyvale, $\mathrm{CA}$ ) or Getting $5 \mathrm{~A}$ amplifier. Extracellular recordings were made by suctioning buccal nerves (BNs) into suction electrodes manufactured from polyethylene tubing and connected to a differential alternating current amplifier model 1700 (A-M Systems, Carlsborg, WA). Data were acquired at $5 \mathrm{kHz}$ with the Digidata 1322A data acquisition system (Molecular Devices) and recorded on a Dell personal computer (Dell Computer Company, Round Rock, TX). Digitized recordings were acquired and analyzed using Axoscope version 9 and Clampfit version 9 (Molecular Devices). A hard copy of electrophysiological recordings was obtained using a chart recorder (MT9500; Astro-Med, West Warwick, RI).

Stimulation paradigms. Neurons were identified based on location, size, and electrophysiological and morphological characteristics as described previously (Hurwitz and Susswein, 1996; Jing et al., 2003). Action potentials in the cerebrobuccal interneuron 2 (CBI-2) were elicited by injecting short direct current (DC) pulses generated by a model S48 stimulator (Grass Instruments, Quincy, MA). To prevent activation of
CBI-3 via electrical coupling, we did not use a concurrent DC depolarization of CBI-2 (Morgan et al., 2002). Motor programs were elicited by a 9-10 Hz stimulation of CBI-2. Each current pulse of $10-20 \mathrm{~ms}$ in duration was set to trigger one action potential. Interstimulation intervals of different durations were used: the short, $30 \mathrm{~s}$ interstimulation interval (30-s-ISI) and the long, $120 \mathrm{~s}$ interstimulation interval (120-sISI). In both paradigms, CBI-2 was stimulated for the duration of the protraction phase, i.e., we terminated the stimulation of CBI- 2 once the protraction phase ended and the retraction phase initiated. Thus, the duration of CBI- 2 stimulation was determined by the duration of the protraction phase rather than the duration of the protraction phase being determined by the duration of CBI-2 stimulation. In the 30-s-ISI paradigm, CBI-2 was not stimulated for a $30 \mathrm{~s}$ time period that followed the end of protraction and then resumed to trigger the next program. In the 120-s-ISI paradigm, a $120 \mathrm{~s}$ CBI-2 interstimulation interval was used.

To elicit individual cycles of egestive programs, unless otherwise indicated, the esophageal nerve (EN) was stimulated every $60 \mathrm{~s}$ with $5 \mathrm{~Hz}, 3$ $\mathrm{ms}$ trains of current pulses for $5 \mathrm{~s}$. In biasing experiments (see Fig. 11), EN was stimulated continuously at $2-3 \mathrm{~Hz}$ with $3 \mathrm{~ms}$ current pulses, the amplitude of which was adjusted to elicit $8-10$ motor programs within a 5 min stimulation period. The $S 48$ stimulator was used to generate current pulses that were injected into the extracellular polyethylene suction electrode into which the EN had been aspirated.

B64 spike timing and motor program classification. The feeding CPG generates several types of motor programs. The major components of these programs are the ones that generate the protraction/retraction and the opening/closing movements of the radula. Independent of the type of output that the CPG generates, the protraction/retraction components occur in a fixed sequence in which the protraction phase precedes the retraction phase (Fig. $1 A$ ). The protraction phase is initiated when protraction-phase interneuron B63 (Fig. $1 B$ ) begins to fire (Hurwitz et al., 1997, 2003; Dembrow et al., 2004). B63 drives protraction neurons, including motoneurons B31/32 and B61/62 (Hurwitz et al., 1996, 2000), and elicits fast IPSPs in the retraction-phase interneuron B64. Neuron B64 fires during retraction and inhibits interneuron B63 and a number of protraction motoneurons, e.g., B31/32 and B61/62. Because neurons B63 and $\mathrm{B} 64$ are on opposite sides of the buccal ganglia, the protraction phase (Fig. $1 \mathrm{~A}$, open bar) was monitored by the activity of the $\mathrm{I} 2$ nerve, which contains axons of protraction motoneurons B31/32 and B61/62 (Hurwitz et al., 1996) that are inhibited by B64. The retraction phase was monitored (Fig. $1 \mathrm{~A}$, filled bar) by activity in BN2 after the termination of 
protraction (Morton and Chiel, 1993) and/or by the sustained depolarization/firing of the plateau-generating interneuron B64 (Hurwitz and Susswein, 1996; Jing et al., 2003; Hurwitz et al., 2005; Koh and Weiss, 2007).

Consistent with other studies in the field (Hurwitz et al., 1996; Nargeot et al., 1997), we operationally defined the time at which the protraction phase ended as the time at which the last spike was recorded in the I2 nerve. Because activity of the bilateral B64s is typically not completely synchronous, we monitored activity of both B64s. Data analysis of B64 firing always refers to the B64 neuron that was the first one to fire an action potential. B64 latency was defined by the timing of the last spike in the I2 nerve relative to the first action potential observed in either of the two B64s. Therefore, B64 latency is referred to as positive when the first B64 action potential follows the last spike in the I2 nerve and negative when the first B64 action potential precedes the last spike in the I2 nerve. In all of the experiments that were performed to test the effects of B64 hyperpolarization on protraction duration, B64s were hyperpolarized bilaterally. The ipsilaterality and contralaterality for B64 was defined with respect to the side on which CBI-2 or EN were stimulated. For instance, if the left CBI-2 or EN was stimulated, the left B64 was defined as the ipsilateral and the right B64 as the contralateral. Notice that in terms of the activity of the I 2 nerve, which was used as the protraction monitor, there is no issue of laterality as the $\mathrm{I} 2$ nerve emerges as a single nerve. We recorded from the $\mathrm{I} 2$ nerve before it reached the I 2 muscle in which the branching occurs. Thus, in effect, the recordings from the I2 nerve are bilateral.

In contrast to the phase-fixed characteristics of the protraction/retraction sequence in each cycle of a motor program, the timing of activity of the radula closing depends on the type of program being generated. In free-feeding animals (Morton and Chiel, 1993; Jing et al., 2007) when the radula closure motoneuron $\mathrm{B} 8$ fires predominantly during retraction and thus helps bring the food into the buccal cavity, the program is considered to be ingestive. When B8 fires predominantly during protraction, and thus helps in removing a nonedible object from the buccal cavity, the program is considered to be egestive. When $\mathrm{B} 8$ firing is similar during protraction and retraction and therefore cannot support either ingestion or egestion, the program is considered intermediate. Based on the recordings obtained in intact feeding animals that showed how patterns of firing of radula closure motoneuron B8 differ in various behaviors, Morgan et al. (2002) defined the criteria for classifying different types of fictive motor programs that are generated by the feeding CPG in the isolated nervous system. Examples of the three types of motor programs are shown in Figure $1 A$. Figure $1 A 1$ is an example of an ingestive program in which $\mathrm{B} 8$ fires predominantly during retraction. Figure $1 A 2$ is an example of an egestive program in which B8 fires predominantly during protraction. Figure $1 A 3$ is an example of an intermediate program in which $\mathrm{B} 8$ firing is similar during both protraction and retraction. For the purposes of this study, we used the previous classification scheme (Morgan et al., 2002) to operationally assign specific motor programs to the ingestive, egestive, and intermediate categories. The boundaries of the ingestive and egestive clusters as defined previously (Morgan et al., 2002) are shown in Figure 1C. The intermediate programs are those that are outside the boundaries that define the ingestive and egestive programs.

Data analysis. Two-group statistical comparisons were performed using $t$ test statistics. Data with more than two groups were first analyzed using one-way ANOVA and thus determine the significance of the overall differences among these groups. Subsequent comparisons between pairs of groups were made using $t$ tests with Bonferroni's corrections. The significance level was set at $p<0.05$. All statistical tests were performed using GraphPad (San Diego, CA) Prism 4.0.

\section{Results}

B64 hyperpolarization in ingestive versus egestive programs

To characterize the contribution that B64 makes to the termination of the protraction phase in the ingestive and the egestive motor programs, we stimulated two inputs to the buccal ganglia, the command-like neuron CBI-2 and the EN (Rosen et al., 1991;
Jing et al., 2004, 2007; Proekt et al., 2004, 2007). Repetitive 30-sISI (see Materials and Methods) stimulation of CBI-2 promotes the generation of ingestive motor programs, whereas stimulation of EN promotes the generation of egestive motor programs. Notably, in semi-intact preparations, the two CPG inputs CBI-2 and $\mathrm{EN}$, respectively, elicit ingestive and egestive behaviors (Chiel et al., 1988; Jing and Weiss, 2005; Zhurov et al., 2005).

CBI-2 was stimulated for the duration of the protraction phase, i.e., we terminated the stimulation of CBI- 2 once the protraction phase ended and the retraction phase initiated. Thus, the duration of CBI-2 stimulation was determined by the duration of the protraction phase rather than the duration of the protraction phase being determined by the duration of CBI-2 stimulation. To study the contribution of $\mathrm{B} 64$ to the termination of the protraction phase of CBI-2-elicited ingestive programs, we compared the protraction duration when the membrane potential of B64 was not manipulated with the protraction duration when B64 was hyperpolarized to prevent or delay B64 firing. B64 was hyperpolarized by injections of $10 \mathrm{nA}$ DC currents. 30-s-ISI stimulation of CBI-2 was used. Figure $2 \mathrm{~A}$ illustrates the results of an individual experiment in which we studied the effects of B64 hyperpolarization on the duration of the protraction phase. In the left panel, B64 membrane potential was not manipulated. In the middle panel, B64 was hyperpolarized. In the right panel, B64 membrane potential was returned to its resting potential. When B64 was hyperpolarized, protraction duration was longer than it was without B64 hyperpolarization. In 21 preparations, compared with control conditions (before and after B64 hyperpolarization), B64 hyperpolarization increased the duration of the protraction phase by $63.71 \%$. Overall, the mean protraction duration in programs elicited before B64 hyperpolarization was $21.33 \pm 1.97 \mathrm{~s}$, in programs in which B64 was hyperpolarized, the protraction duration was $34.92 \pm 1.95 \mathrm{~s}$, and in programs elicited after B64 hyperpolarization, the protraction duration was $21.51 \pm 2.32 \mathrm{~s}$ (Fig. 2 B). Statistical analyses showed that there was a significant overall difference between the three conditions $\left(F_{(2,40)}=29.56 ; p<0.0001 ; n=21\right)$.

Similar results were obtained in the preparations in which repetitive ingestive responses were elicited through a continuous stimulation of CBI-2 (Hurwitz et al., 2005). Figure $2 C$ illustrates the experiment in which bilateral hyperpolarization of B64 extended the duration of the protraction phase for the duration of hyperpolarization. Average protraction duration during B64 hyperpolarization (26.48 $\pm 3.07 \mathrm{~s})$ was significantly longer $(p<$ $0.05 ; t=4.30$, paired $t$ test; $n=4$ ) compared with controls (8.65 $\pm 1.27 \mathrm{~s})$. In addition, average cycle period during B64 hyperpolarization $(42.78 \pm 9.30 \mathrm{~s})$ was also significantly longer $(p<0.05 ; t=3.29$, paired $t$ test; $n=4)$ compared with controls $(18.30 \pm 1.87 \mathrm{~s})$. This also delayed the time at which subsequent programs were initiated. Notably, this shift persisted even after the activity of B64 was no longer manipulated. These results support the idea that B64 firing plays an important role in terminating the protraction phase of CBI-2-elicited ingestive motor programs and that it does so independent of whether single or repetitive motor programs are triggered.

We next studied the effects of B64 hyperpolarization on ENelicited egestive motor programs. Figure $3 A$ illustrates the results of an experiment in which we examined the effects of B64 hyperpolarization on the duration of the protraction phase. In the left panel, B64 membrane potential was not manipulated. In the middle panel, B64 was hyperpolarized. In the right panel, B64 was returned to its resting membrane potential. Unlike in the case of CBI-2-elicited programs, B64 hyperpolarization did not extend 


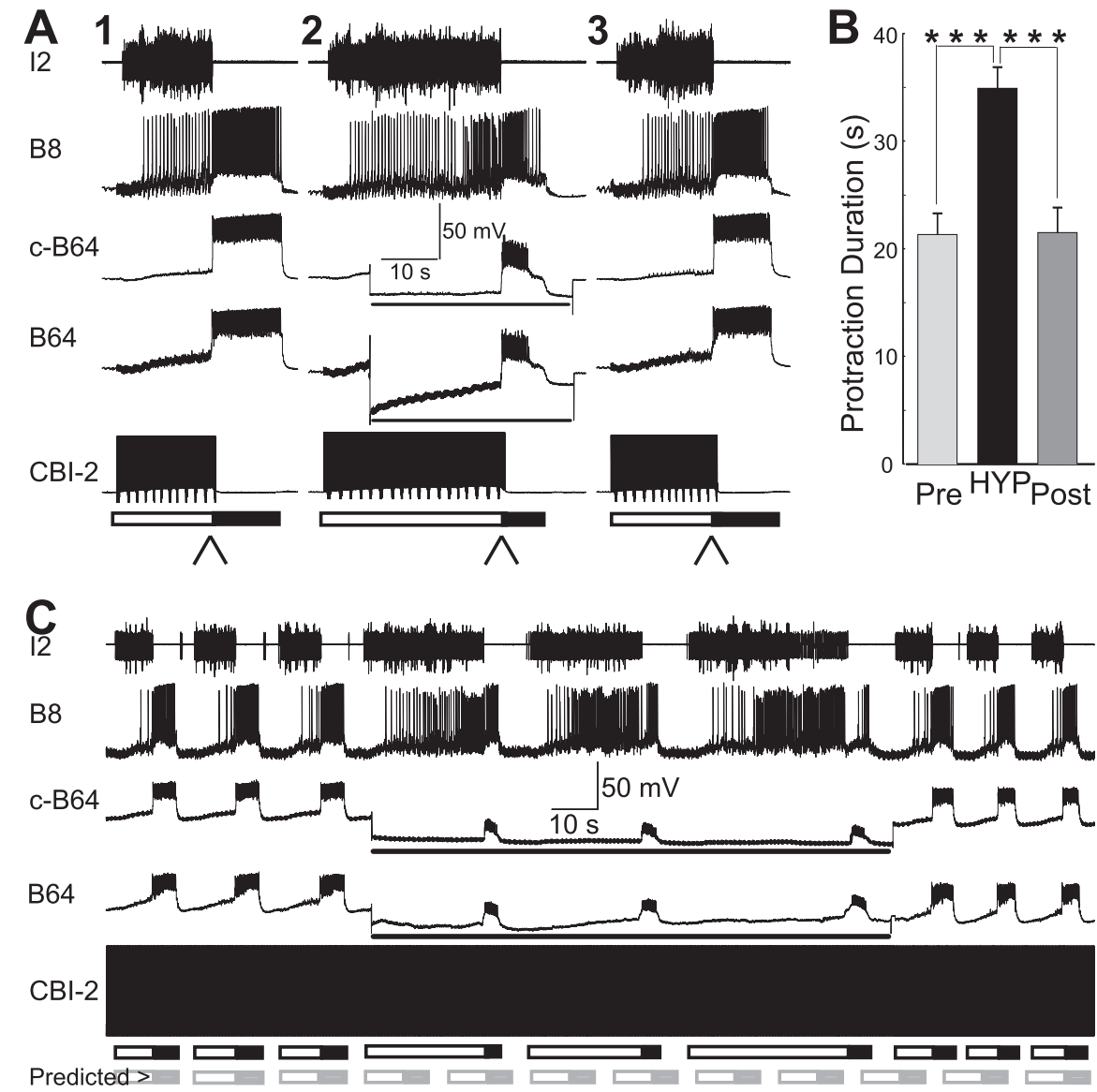

Figure 2. Bilateral hyperpolarization of $\mathrm{B} 64$ lengthens the protraction duration of $(\mathrm{BI}-2$-elicited ingestive motor programs. $\boldsymbol{A}$, Illustrative examples. A single cycle of an ingestive motor program was elicited by $10 \mathrm{~Hz}$ CBI-2 stimulation (A1). A second ingestive motor program was elicited by $(B \mathrm{l}-2$ in which both $B 64$ s were hyperpolarized (lines below the B64 traces) with $10 \mathrm{nA}$ current $(\boldsymbol{A 2})$. A third ingestive program was elicited without B64 hyperpolarization (A3). Protraction duration was longer when B64 was hyperpolarized. The arrowheads below the bars denote the phase transition from protraction to retraction. $\boldsymbol{B}, \mathrm{Grouped}$ data show that protraction duration was significantly longer after both B64s were hyperpolarized. Pre, The first ingestive program induced by $\mathrm{CBI}-2$; HYP, a program induced by $\mathrm{CBI}-2$ with hyperpolarization of both $\mathrm{B} 64 \mathrm{~s}$; Post, a recovery program induced by $\mathrm{CBI}-2$. Post hoc tests with Bonferroni's corrections, ${ }^{* * *} p<0.001$. Error bars indicate SEM. C, An example in which multiple cycles of ingestive motor programs were elicited through a continuous stimulation of CBI-2 illustrates similar extending effects of B64 hyperpolarization on protraction duration. This paradigm also demonstrates that cycle periods were extended when B64s were hyperpolarized. The gray bars at the bottom show predicted protraction and retraction had B64 not been hyperpolarized, illustrating the effects of B64 hyperpolarization on protraction duration and cycle periods. In this figure, and all subsequent figures in which $\mathrm{CBI}-2$ stimulation was used to elicit motor programs, C-B64 refers to the B64 that is contralateral to the stimulated CBI-2. In cases in which EN was stimulated to elicit motor programs, C-B64 refers to B64 that was contralateral to the stimulated EN. programs (controls, $16.25 \pm 0.75$ s vs B64 hyperpolarization, $16.47 \pm 0.35 \mathrm{~s} ; \mathrm{p}=$ $0.79 ; t=0.28$, paired $t$ test $)$.

We considered the possibility that B64 hyperpolarization might have been ineffective if $\mathrm{B} 64$ action potentials were generated distally from the soma (Hurwitz and Susswein, 1996) and/or if generation of egestive programs was accompanied by a significant increase in conductance of the part of the axon that separates the soma from the spike initiation zone. This is unlikely to be the case because, even when the injected current was increased from 10 to $30 \mathrm{nA}$, protraction duration was not extended (data not shown). These experiments indicate that, although B64 is active in EN-elicited egestive programs, unlike in CBI-2-stimulation-elicited ingestive programs, B64 activity is not responsible for the termination of the protraction phase at the time at which it is normally terminated.

\section{B64 early activation in ingestive versus} egestive programs

The observation that $\mathrm{B} 64$ is active in both ingestive and egestive programs yet its hyperpolarization affects protraction duration in CBI-2 ingestive but not EN egestive motor programs raised the possibility that the inhibitory output of B64 may be selectively gated out in EN-stimulation-elicited egestive programs. Notably, precedents for such gating out exist in the feeding system of Aplysia (Weiss et al., 1986; Evans et al., 2003; Hurwitz et al., 2005) as well as in the crab (Bartos and Nusbaum, 1997). Thus, we tested the possibility that the output of B64 is functional in CBI-2-elicited ingestive programs but is gated out in ENelicited egestive programs.

In eight preparations, ingestive programs were elicited by 30 -s-ISI CBI-2 stimulation. Injection of brief DC current the duration of the protraction phase in EN-elicited egestive programs. Overall, the mean protraction duration in programs elicited before B64 hyperpolarization was $11.97 \pm 1.10 \mathrm{~s}$, in programs in which B64 was hyperpolarized, the protraction duration was $11.93 \pm 1.01 \mathrm{~s}$, and in programs elicited after B64 was returned to its resting potential, the protraction duration was $12.12 \pm 0.97 \mathrm{~s}(n=9)$ (Fig. $3 B)$. Statistical analyses showed that there was no significant overall difference between the three conditions $\left(F_{(2,16)}=0.36 ; p=0.71\right)$. Similar results were obtained when repetitive egestive motor programs were elicited through a continuous stimulation of EN $(n=6)$. Figure $3 C$ illustrates the experiment in which EN elicited multiple-cycle motor programs and B64s were hyperpolarized for the duration of three cycles. Similar to what we observed when single programs were elicited, hyperpolarization of B64 did not affect the duration of the protraction phase (controls, $6.71 \pm 0.50 \mathrm{~s}$ vs $\mathrm{B} 64$ hyperpolarization, $6.95 \pm 0.49 \mathrm{~s} ; p=0.61 ; t=0.56$, paired $t$ test). In addition, B64 hyperpolarization did not alter the cycle period of the motor pulses in the early part of the protraction phase was used to activate B64. The results of such an experiment are shown in Figure $4 \mathrm{~A}$. In the left panel, the firing of B64 was not controlled, i.e., B64 was allowed to fire on its own, in the middle panel, B64 was fired prematurely via a brief DC current injection during the protraction phase, and in the right panel, the B64 was again allowed to fire on its own. The protraction phase was terminated immediately when B64 was fired prematurely and therefore the protraction phase was shortened. This was the case in all eight preparations in which we fired B64 early in the protraction phase. We also sought to determine whether, in repetitive motor programs elicited by a continuous stimulation of CBI-2, a premature firing of B64 could shorten the duration of the protraction phase. We found that this was indeed the case $(n=6)$ (Fig. $4 B)$. This shortening of the duration of protraction happened when either one (single asterisk) or both (two asterisks) B64s were fired prematurely (controls, $18.78 \pm 5.44 \mathrm{~s}$ vs B64 depolarization, $7.81 \pm$ $1.87 \mathrm{~s} ; p<0.05 ; t=3.02$, paired $t$ test). The shortening of the 
duration of the protraction phase also affected the timing of motor programs that were subsequently elicited without manipulating the B64 activity. The two rows of bars on the bottom of Figure $4 B$ illustrate this point. The top row of bars indicates the actual protraction and retraction phase of each motor program. The bottom row of bars indicates the predicted timing of protraction and retractions if the premature termination of the protraction phase had not been elicited via stimulation of B64. The arrows point out the shifts between the predicted and the actual onset of protractions. This effect is reflected as a shortening of cycle period (controls, $32.59 \pm 7.69$ s vs B64 depolarization, $14.16 \pm 2.71 \mathrm{~s} ; p<0.05 ; t=3.54$, paired $t$ test). Thus, independent of whether single or repetitive motor programs are elicited via stimulation of $\mathrm{CBI}-2$, premature firing of $\mathrm{B} 64$ shortens the duration of the protraction phase. These results are consistent with previous work on uncharacterized CBI-2-elicited motor programs (Hurwitz et al., 2005).

We performed similar experiments in the EN-stimulation-elicited egestive programs. Figure $4 C$ illustrates the results of the experiment in which single cycles of motor programs were elicited. In the left panel, firing of B64 was not controlled, in the middle panel, B64 was fired prematurely in the protraction phase, and in the right panel, the firing of B64 was not controlled. The duration of the protraction phase was shortened when B64 was fired prematurely. This was the case in all seven preparations in which we fired B64 early in the protraction phase. We also investigated the effects of premature firing of B64 on the duration of the protraction phase of repetitive egestive motor programs that were elicited by a continuous EN stimulation (Fig. 4D). In all six preparations in which we conducted this experiment, premature firing of B64 shortened the duration of the protraction phase (controls, $7.49 \pm 0.47 \mathrm{~s}$ vs B64 depolarization, $3.25 \pm 0.19 \mathrm{~s} ; p<0.001 ; t=8.30$, paired $t$ test). The B64stimulation-elicited shortening of the protraction phase did not require a bilateral stimulation of B64. Stimulation of either of the two B64 was sufficient to terminate the protraction phase. This may explain (see below) why the protraction phase of motor programs is terminated even when the activity of only one B64 is initiated during protraction. Thus, independent of whether single or repetitive motor programs are elicited via stimulation of $\mathrm{EN}$, premature firing of $\mathrm{B} 64$ shortens the duration of the protraction phase. Also, as was the case for the repetitive ingestive programs, premature firing of B64 advanced the onset of motor programs that were subsequently elicited, whereas the B64 activity was not manipulated (Fig. 4, see the two rows of bars at the bottom of $D$ and the explanation provided in the description of $B)$. Again, this effect is reflected as a shortening of cycle period (controls, $18.11 \pm 0.91 \mathrm{~s}$ vs B64 depolarization, $11.30 \pm 0.82 \mathrm{~s}$; $p<0.05 ; t=6.02$, paired $t$ test). The fact that $\mathrm{B} 64$ can prema- turely terminate the protraction phase of both CBI-2stimulation-elicited ingestive and EN-stimulation-elicited egestive programs indicates that the inhibitory output of B64 is not differentially gated in the ingestive versus egestive motor programs and therefore cannot account for the differential effects of B64 hyperpolarization experiments shown in Figures 2 and 3.

\section{Timing of B64 activity}

B64 was active in both ingestive and egestive motor programs, and differential gating of B64 did not appear to explain the differential effects of B64 hyperpolarization. Furthermore, hyperpolarization experiments (Figs. 2, 3) suggested the existence of other, B64-independent means of protraction termination. Therefore, we reasoned that, in EN-stimulation-elicited egestive programs, these B64-independent means of protraction termination may come into play before $\mathrm{B} 64$ begins to fire. If this were the case, B64 might be expected to begin to fire only after the protraction phase of the EN-elicited-egestive programs was already terminated. Because under these conditions the protraction phase would have already been terminated when B64 firing occurred, there would be no protraction phase to be terminated by B64 and therefore not only would B64 not act as a protraction 

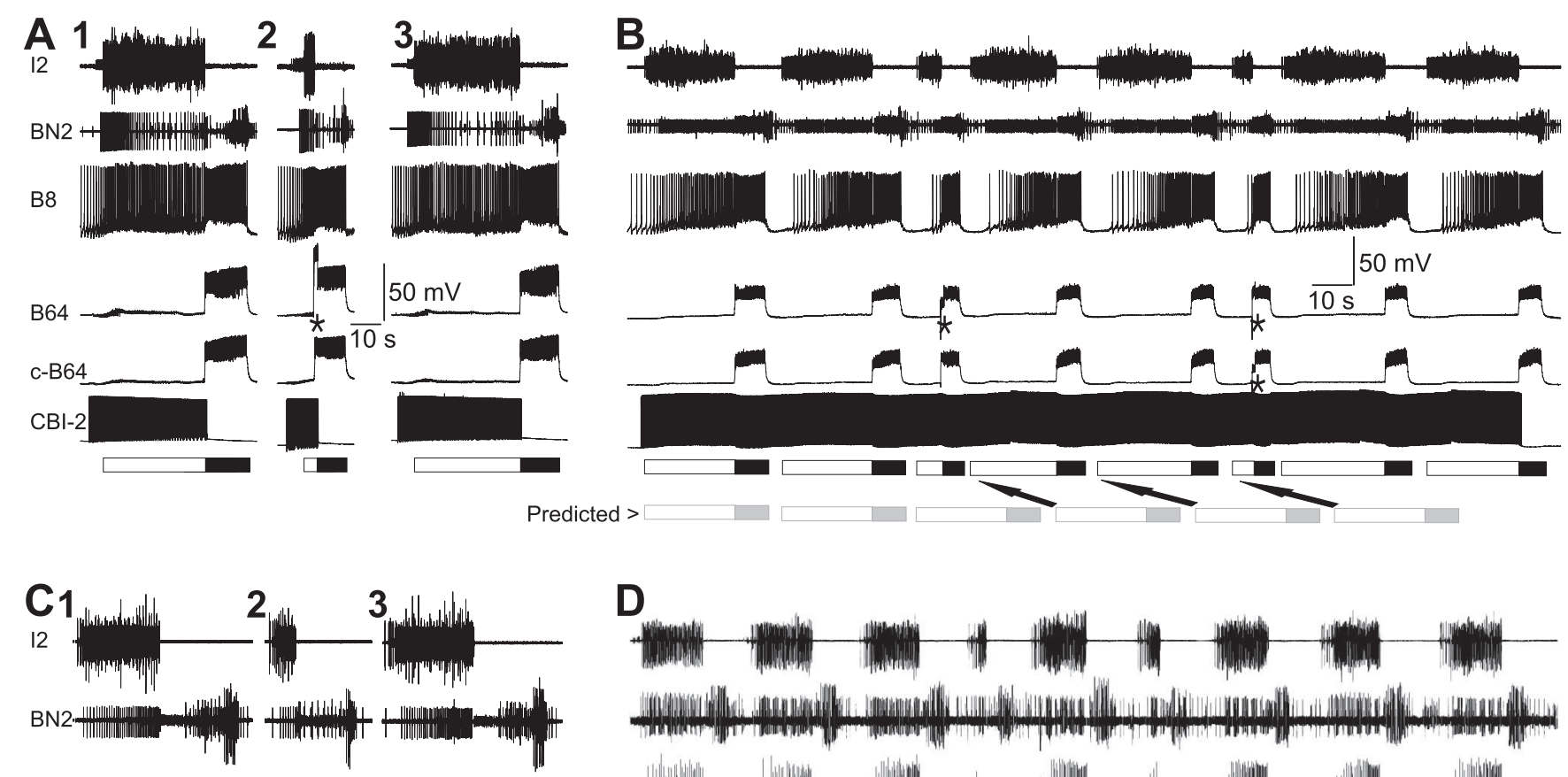

D

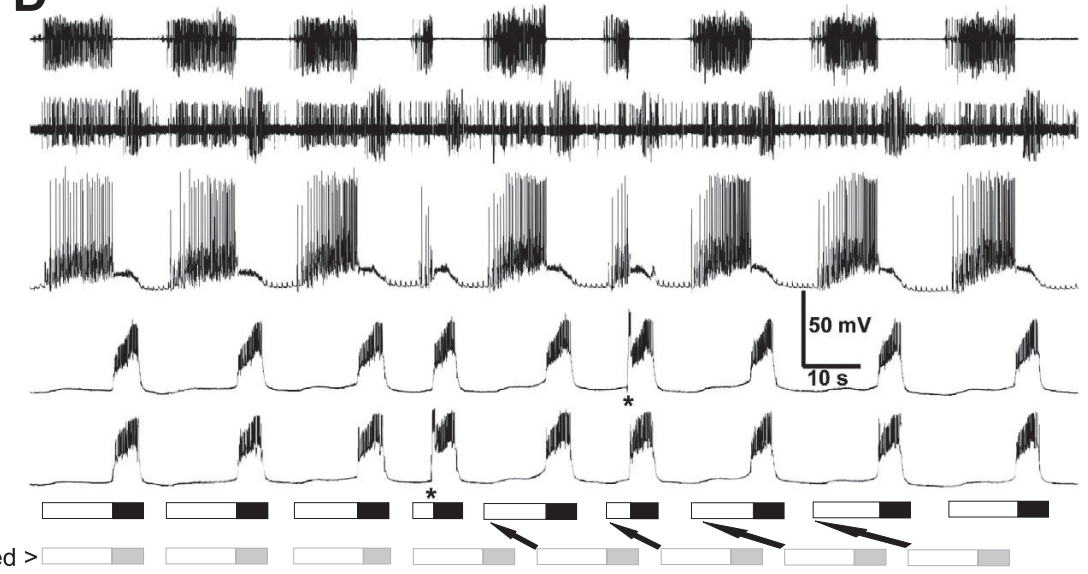

Predicted $>$

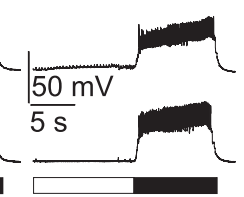

Figure 4. Early activation of $B 64$ prematurely terminates protraction and initiates retraction in both $C B I-2$-elicited ingestive and EN-elicited egestive programs. $A$, Firing one B64 prematurely terminates the protraction phase in (BI-2-elicited ingestive programs. A single cycle of an ingestive motor program was elicited by $\mathrm{CBI} 2$ ( $\mathrm{A}$ 1). When a brief depolarizing current was injected into one B64 $\left(^{*}\right)$ seconds before retraction was expected to occur, the protraction phase was prematurely terminated and a full retraction phase was initiated (A2). Another ingestive program was elicited without current injection into B64 (A3), which had a similar protraction duration as that in $\mathbf{A 1}$. B, An example in which multiple cycles of ingestive motor programs were elicited through a continuous stimulation of $\mathrm{CBI}-2$ illustrates similar shortening effects of $\mathrm{B} 64$ depolarization $\left(^{*}\right)$ on protraction duration. This paradigm also demonstrates that cycle periods were shortened when B64s were depolarized. C, Firing one B64 also prematurely terminates the protraction phase in $\mathrm{EN}$-elicited egestive programs. A single cycle of an egestive motor program was elicited by stimulation of the $\mathrm{EN}$ (C1). When a depolarizing current was injected into one B64 $\left.{ }^{*}\right)$ seconds before retraction was expected to occur, the protraction phase was prematurely terminated and a full retraction phase was initiated (C2). Another egestive program was elicited without current injection into B64 (C3). D, An example in which multiple cycles of egestive motor programs were elicited through a continuous stimulation of EN illustrates similar shortening effects of B64 depolarization $\left(^{*}\right)$ on protraction duration. This paradigm also demonstrates that cycle periods were shortened when B64 was depolarized. The gray bars at the bottom of $\boldsymbol{B}$ and $\boldsymbol{D}$ show predicted protraction and retraction had $\mathrm{B} 64$ not been depolarized, illustrating the effects of B64 depolarization on protraction duration and cycle periods.

terminator, but also B64 hyperpolarization would not be able to extend the duration of the protraction phase.

The activity of the two B64 neurons is not completely synchronous. This raises the possibility that the termination of the protraction phase depends on the average activity of the two B64s rather on a single B64. However, examination of numerous records (see Figs. $6 A 1, A 3,7 B 1,8 B 1, B 3$ ) showed that the firing of the leading B64 sufficed to terminate the protraction phase and that protraction commonly terminated after the onset firing of the leading B64 but before the onset firing of the second B64. This strongly suggests that the firing of the leading B64 may be sufficient to terminate the protraction phase (compare Fig. 4). Therefore, to quantify the temporal relationship between the onset of B64 firing and the termination of the protraction phase (end of spike activity in the I2 nerve), we defined B64 latency as the time that elapsed between the last spike recorded in the I2 nerve and the first action potential that was recorded in either of the two B64s. When the first B64 action potential occurred after the last spike in I2, the latency was defined as positive. When the first action potential in B64 occurred before the last spike in I2, B64 latency was defined as negative. Data for B64 latency were derived from experiments shown in Figures 2 and 3. We analyzed 47 CBI-2-elicited ingestive motor programs from 18 preparations, and an example from a single preparation (Fig. $5 \mathrm{~A}$ ) shows that the initiation of $\mathrm{B} 64$ firing preceded the termination of the protraction phase. Grouped data show that the mean latency of B64 was negative $(-54.00 \pm 13.31 \mathrm{~ms})$. We also analyzed 50 egestive programs from 16 preparations in which egestive programs were elicited by EN stimulation. In contrast to the negative latencies recorded in CBI-2-stimulation-elicited ingestive programs, B64 latencies of EN-stimulation-elicited egestive programs were positive ( $126.70 \pm 14.10 \mathrm{~ms})$. Figure $5 B$ illustrates the firing of B64 in an egestive program. Overall, there was a significant difference $(p<0.001)$ between the B64 latencies recorded in the two types of motor programs, with the mean latencies recorded in ingestive programs being negative ( 41 of the total of 47 programs) and the 


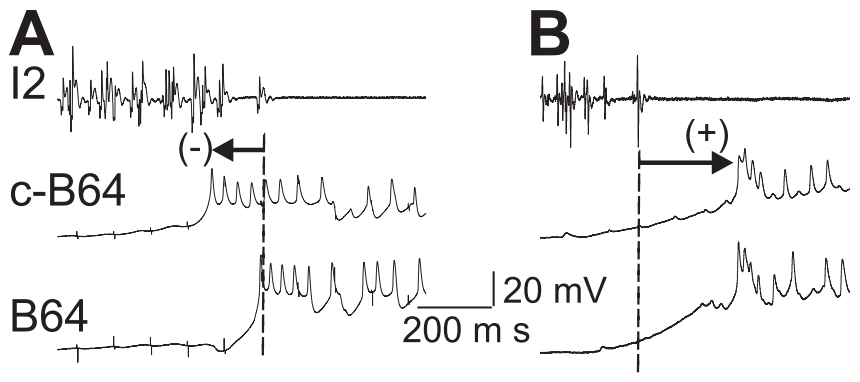

Figure 5. Different $\mathrm{B} 64$ latency in $\mathrm{CBI}-2$-elicited ingestive programs versus $\mathrm{EN}$-elicited egestive programs. $A, B 64$ latency was negative in an ingestive program elicited by $C B \mathrm{~B}-2$ because B64 spiking was initiated before the last spike in 12 nerve. $\boldsymbol{B}, \mathrm{B} 64$ latency was positive in an egestive program induced by EN stimulation because B64 spiking was initiated after the last spike in the 12 nerve. Vertical broken lines in this and subsequent figures denote the timing of the last spike in the 12 nerve, which was operationally defined as the end of protraction.

mean latencies recorded in egestive programs being positive (48 of 50 programs).

Consistent with visual observations, we found no significant difference in the phasing of the sustained firing of neuron B64 in ingestive vs. egestive programs. Specifically, we sought to define the retraction-phase duration independently of B64 firing by measuring the time that elapsed between the last I 2 spike and the last spike in the high-frequency burst in buccal nerve 2 (Morton and Chiel, 1993) (see Materials and Methods); therefore, we analyzed the data from preparations in which we recorded both the activity of BN2 and bilateral B64s (for examples, see Fig. $1 \mathrm{~A}$ ). We found that there was no significant difference $(p=0.91 ; t=0.11)$ between the fraction of retraction duration during which B64 fired in ingestive programs $(84.43 \pm 2.54 \%)(n=4)$ verses that during egestive programs $(84.79 \pm 1.98 \%)(n=7)$. Overall, the duration of B64 firing during retraction accounted for $100 \%$ of the total duration of B64 firing in egestive programs $(n=7)$ and for $97.99 \%(n=4)$ of the total duration of B64 firing in ingestive programs. Thus, the major difference in terms of phasing of the sustained activity of B64 appears to be that B64 firing was initiated earlier in the ingestive programs than it was in the egestive programs.

Together, our data indicate that it is the timing of the onset of B64 firing that may play a major role in determining whether B64 acts as a protraction terminator. Not surprisingly, in terms of the ability of B64 firing to terminate the protraction phase, when B64 firing is initiated before the termination of protraction phase, B64 acts as the protraction terminator. When B64 firing is initiated after the protraction phase is terminated, the termination is accomplished by B64-independent means. These results suggest the existence of at least two means of protraction termination, the B64-dependent and the B64-independent means. These two means appear to be preferentially used in different types of motor programs.

\section{B64 latency in hyperpolarization experiments}

We further probed the contribution of B64-dependent and B64independent means of protraction-phase termination by examining B64 latency in hyperpolarization experiments. The middle panel of Figure $2 \mathrm{~A}$ shows that, even when B64 is hyperpolarized, B64 breaks out of hyperpolarization and begins to fire at the end of the protraction phase. In principle, this delayed B64 firing, rather than some other B64-independent means, could potentially function to terminate the protraction phase albeit with a delay attributable to B64 hyperpolarization. If this were the case, we would expect that, even with B64 hyperpolarized, B64 latencies of CBI-2-elicited ingestive programs would remain negative. After we analyzed B64 latency data from experiments shown in Figure $2 A$, we found that this was not the case. Under control conditions, i.e., before and after B64 hyperpolarization (Fig. $6 A 1, A 3), \mathrm{B} 64$ fired with negative latencies relative to the termination of spike activity in the 12 nerve. However, the situation changed when B64 was hyperpolarized and the protractionphase termination was delayed. Under these conditions, B64 latency became positive (Fig. 6A2). The statistical analysis of the grouped data $(n=21)$ (Fig. $6 B)$ revealed a significant overall difference between B64 latencies under the three conditions $\left(F_{(2,50)}=92.38 ; p<0.0001\right)$. Importantly, B64 latencies measured during B64 hyperpolarization were positive. Apparently when B64 was hyperpolarized, it no longer acted as a protractionphase terminator and the protraction phase appeared to be terminated in a B64-independent manner. These results suggest that, in CBI-2-elicited ingestive programs, B64 may be necessary for the termination of the protraction phase under normal conditions, but that B64-independent means may be deployed to terminate the protraction phase when B64 is hyperpolarized.

\section{B64 latency in different types of motor programs}

In the above experiments, we characterized the contribution of B64 to protraction termination in the ingestive versus egestive motor programs. However, in these experiments, the two types of motor programs were elicited by different inputs, i.e., the ingestive programs were elicited by stimulation of CBI-2, whereas the egestive ones were elicited by stimulation of the esophageal nerve. This raised the question whether the positive versus negative latency of B64 firing was related to the nature of the elicited programs or to the specific input that was used to elicit the programs. To gain insight into this problem, we took advantage of the previous work that showed that, when infrequently stimulated (ISI $>90$ s, i.e., 120 -s-ISI), CBI-2 predominantly elicits intermediate programs but in a smaller number of preparations can elicit either ingestive responses and in other preparations can elicit egestive responses (Fig. 1A) (Jing and Weiss, 2001, 2002; Morgan et al., 2002; Proekt et al., 2004). With this 120-s-ISI stimulation paradigm, the programs tend to maintain stable characteristics and thus appear to reflect the resting steady state of the preparation. We reasoned that, if it is the CBI- 2 input that determines the positive versus the negative latencies of B64 firing, then B64 latencies would be negative independent of whether the CBI-2elicited program is ingestive, egestive, or intermediate. Alternatively, if it is the nature of the motor program, rather than the CBI-2 input, that determines whether B64 latencies are positive or negative, then in CBI-2-elicited ingestive programs, B64 latency should be negative, whereas in CBI-2-elicited egestive programs, the latency should be positive.

We analyzed 246 programs from 50 preparations. Programs were elicited using the 120-s-ISI CBI-2 stimulation (Fig. 7A) (see Materials and Methods). Sixty-one of these programs were classified (see Materials and Methods) as ingestive, 113 as intermediate, and 72 as egestive. To illustrate the latencies of B64 firing, Figure $7 B$ shows, with a faster time base, the transition period between the protraction and retraction phases of each of the programs shown in Figure $7 A$. We found that, in the programs classified as ingestive (Fig. 7A1), 87.33\% of B64 latencies were negative, and $12.67 \%$ were positive. In the programs classified as egestive (Fig. 7A4), 86.74\% of B64 latencies were positive, and $13.26 \%$ were negative. In programs classified as intermediate, $54.31 \%$ of B64 latencies were negative (Fig. 7A2), and $46.69 \%$ 


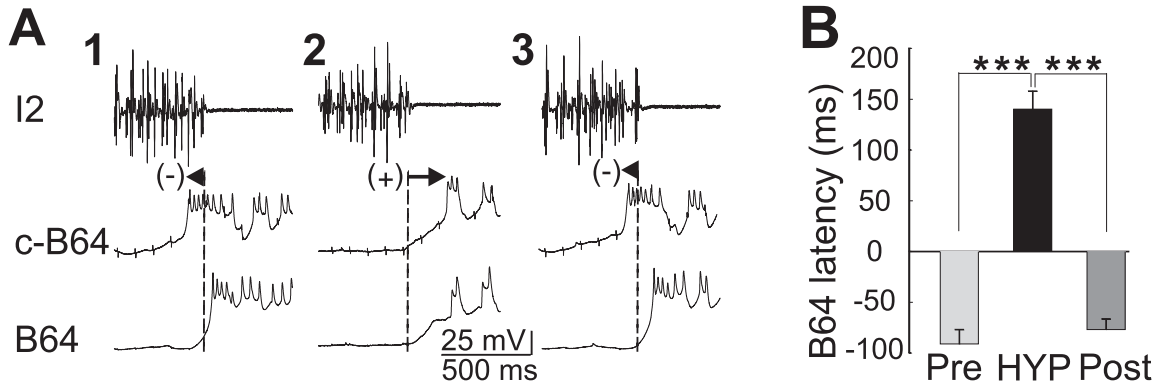

Figure 6. Shift of $B 64$ latency during $B 64$ hyperpolarization experiments in $C B 1-2$-elicited ingestive programs. $A$, Expanded

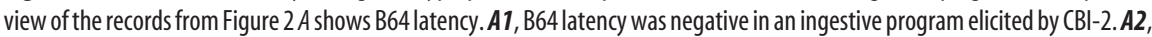
Bilateral hyperpolarization of $B 64$ s delayed onset of $B 64$ firing relative to the last spike in the 12 , and $B 64$ latency now shifted to positive in a $\mathrm{CBI}$-2-elicited ingestive motor program. $\mathbf{A 3}, \mathrm{B} 64$ latency was negative when B64s were not hyperpolarized in the ingestive program elicited by $\mathrm{CBI}-2$. $B$, Grouped data show that there is a significant difference between $B 64$ latency in control conditions (Pre, before B64 hyperpolarization; Post, after B64 hyperpolarization) and that during B64 hyperpolarization (HYP). Post hoc tests with Bonferroni's corrections, ${ }^{* * *} p<0.001$. Error bars indicate SEM.
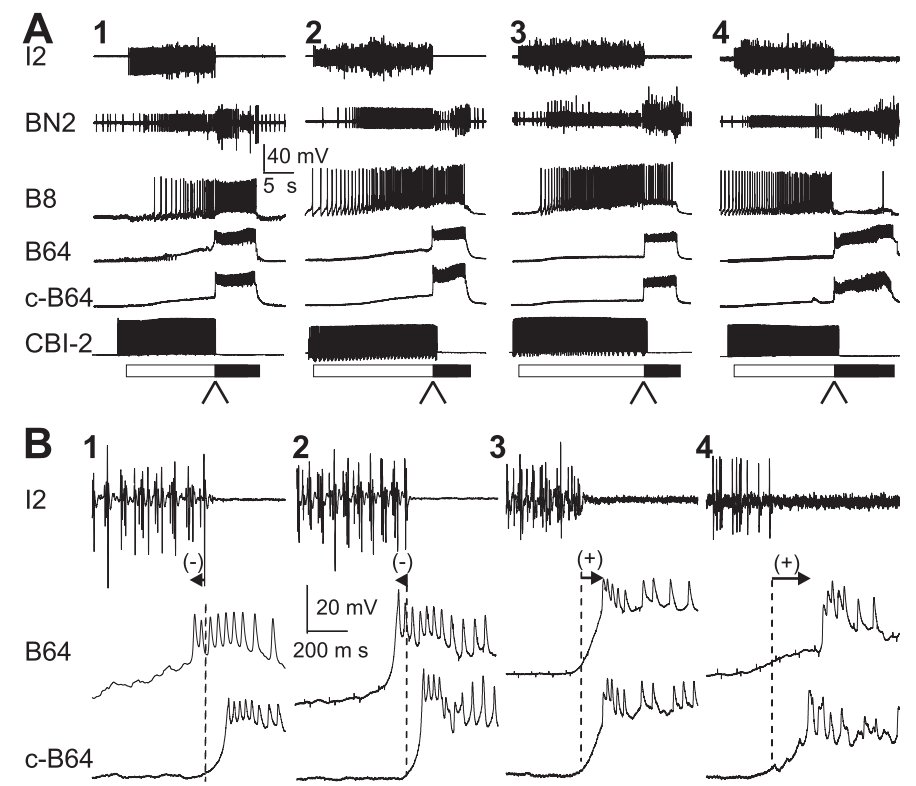

Figure 7. Different $\mathrm{B} 64$ latency in ingestive, intermediate, and egestive programs elicited by $\mathrm{CBI}-2$. Motor programs are elicited by CBI-2 using 120-s-ISI stimulation paradigm. $\boldsymbol{A}$, Illustrative examples. $\boldsymbol{A} 1$, A single cycle of a CBI-2-elicited ingestive motor program. The radula closer motoneuron $B 8$ fired predominantly during the retraction phase. $\mathbf{A 2}, \boldsymbol{A} \mathbf{3}$, Two single cycles of CBI-2-elicited intermediate motor programs. B8 fired at moderate rates during both protraction and retraction. $\mathbf{A 4}$, A single cycle of a CBI-2-elicited egestive motor program. B8 fired vigorously during the protraction phase. The arrowheads below the bars point to the phase transition from protraction to retraction. $\boldsymbol{B}$, Different $B 64$ latency in different types of motor programs elicited by CBI-2. B1-B4, Expanded view of the records from $A$ 1-A4, respectively (at the places where arrowheads are pointing to), shows B64 latency in these different programs. B64 latency was negative in the ingestive program (B1) and positive in the egestive program (B4). In intermediate programs, B64 latency can be either negative (B2) or positive (B3). $\boldsymbol{C}$, Grouped data show a significant difference in $B 64$ latency between ingestive programs and intermediate programs, between intermediate programs and egestive programs, and between ingestive programs and egestive programs. Labels $1-4$ refer to the type of motor programs as illustrated in $\mathbf{A 1 - A 4 , ~ r e s p e c t i v e l y . ~ T h e ~ " 2 \& 3 " ~ g r o u p ~ r e p r e s e n t s ~ i n t e r m e d i a t e ~ p r o g r a m s ~ t h a t ~ w e r e ~ c o m b i n e d ~ i n d e p e n d e n t ~ o f ~}$ whether their latencies were positive or negative. $\boldsymbol{D}$, The intermediate programs are further divided into two groups: ingestivelike (Ing-like) and egestive-like (Ege-like). Ingestive-like are those intermediate programs with higher mean B8 activity during retraction than during protraction, whereas egestive-like are those intermediate programs with higher mean B8 activity during protraction than during retraction. Group data show that B64 latency for ingestive-like programs is negative, whereas for egestive-like programs is positive. Post hoc tests with Bonferroni's corrections, ${ }^{*} p<0.05 ;{ }^{* *} p<0.01 ;{ }^{* *} p<0.001$. Error bars indicate SEM.

were positive (Fig. 7A3). Overall, the mean B64 latency in ingestive programs was $-58.95 \pm 6.03 \mathrm{~ms}$, the mean latency of B64 in egestive programs was $169.20 \pm 14.12 \mathrm{~ms}$, and the mean latency of intermediate programs was $-6.39 \pm 9.12 \mathrm{~ms}$ (Fig. $7 C)$. This difference was statistically significant $\left(F_{(2,250)}=102.41 ; p<\right.$
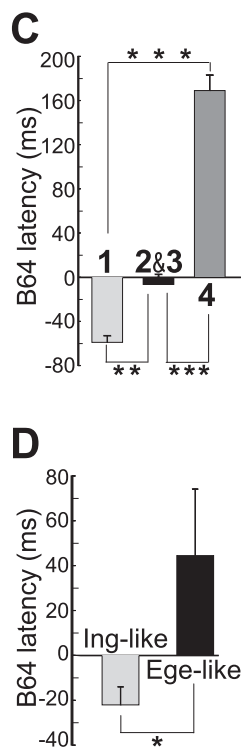

0.0001). Together, comparisons of B64 latencies in CBI-2-elicited ingestive versus egestive programs suggest that the latency of B64 firing, and consequently the ability of B64 to act as a protraction terminator, may be more closely related to the nature of the motor program that the CPG generates than to the specific input that is used to elicit the motor program.

Although motor programs can be classified as ingestive, egestive, and intermediate, there appears to be a continuum of ingestiveness versus egestiveness, in which the ingestive programs are on the one end of the continuum and the egestive programs are at the other end. We explored the possibility that the relationship between the degree of ingestiveness/egestiveness and the latency of B64 firing may already be apparent when the program is classified as intermediate. We therefore classified intermediate programs as "more ingestive-like" when B8 mean firing rate was higher during retraction than during protraction, and "more egestive-like" when B8 mean firing rate was higher during protraction than during retraction. We found that, indeed, the latency of B64 was negative in the intermediate programs classified as more ingestive-like. In contrast, the latency of $\mathrm{B} 64$ was positive in intermediate programs classified as more egestive-like (Fig. $7 D$ ). The difference was statistically significant $(p<0.05)$. These results support the notion that the latency of the onset of B64 firing is related to the degree of ingestiveness versus egestiveness of the motor program even when, based on B8 activity, these programs are classified as intermediate.

\section{B64 latency in intermediate programs}

The observation that B64 latencies of intermediate programs could be either positive or negative suggests that the intermediate programs may not constitute a homogeneous group of programs and that the contribution of B64 to the termination of intermediate programs may be different in those intermediate programs in which the latency of B64 firing is positive than in those intermediate programs in which the latency of B64 firing is negative. Therefore, using the 120-s-ISI CBI-2 stimulation, we compared the effects of B64 hyperpolarization on protraction duration in preparations in which the B64 latencies were positive with those in which they were negative. Figure $8 \mathrm{~A}$ shows an example of such a hyperpolarization experiment from a preparation in which CBI-2 elicited intermediate programs and the B64 latency was negative. The middle panel (A2) shows the program that was elicited when B64 was hyperpolarized. The left $(A 1)$ and the right $(A 3)$ panels show the 
programs elicited, respectively, before and after but without the B64 hyperpolarization. In Figure $8 B$, faster sweeps of parts of traces shown in Figure $8 A$ are plotted to illustrate the transition from the protraction to the retraction phase. Overall, the duration of the protraction phase was $26.57 \pm 2.74 s$ when B64 was hyperpolarized and $14.97 \pm 1.76 \mathrm{~s}$ before the hyperpolarization and $15.26 \pm 2.30 \mathrm{~s}$ after hyperpolarization $(n=10)$ (Fig. $8 C$ ). The overall difference was statistically significant $\left(F_{(2,18)}=10.12 ; p<0.01\right)$. In addition, B64 latency (Fig. $8 D$ ) showed significant differences in the three conditions: "Pre," "HYP," and "Post" $\left(F_{(2,18)}=39.95\right.$; $p<0.001)$. In other words, in these programs, the effect of B64 hyperpolarization on protraction duration and B64 latency was similar to that observed in CBI-2elicited ingestive programs.

Figure $8 E$ shows an example of a B64 hyperpolarization experiment in a preparation in which stimulation of CBI-2 elicited intermediate programs and the B64 latency was positive. The middle panel (E2) shows the program that was elicited when B64 was hyperpolarized, and the left (E1) and right (E3) panels show the programs elicited before and after but without B64 hyperpolarization. In Figure $8 F$, faster sweeps of parts of traces shown in Figure $8 E$ are plotted to illustrate the transition from the protraction to the retraction phase. On average, the duration of the protraction phase was $18.23 \pm 2.60 \mathrm{~s}$ when B64 was hyperpolarized and $18.08 \pm 2.64 \mathrm{~s}$ before hyperpolarization and $17.79 \pm$ $2.59 \mathrm{~s}$ after hyperpolarization $(n=16)$ (Fig. 8G). The overall difference was not statistically significant $\left(F_{(2,30)}=0.34 ; p=\right.$ 0.72). In addition, B64 latency (Fig. $8 H$ ) remained positive in the three conditions ("Pre," "HYP," and "Post") and was more positive when B64s were hyperpolarized $\left(F_{(2,30)}=33.01 ; p<0.001\right)$. Thus, in these programs, the effect of B64 hyperpolarization was similar to that observed in $\mathrm{EN}$ egestive programs in that the duration of the protraction phase was not extended.

To further probe the relationship of B64 latency versus the effects of B64 hyperpolarization, we plotted B64 latency against percentage change of protraction duration for all B64 hyperpolarization experiments (Fig. 9). Consistent with the idea that it is the negative versus the positive timing of B64 latencies that determines whether B64 participates in the termination of the protraction phase of a program, we found that, for the negative latencies, the percentage change of protraction duration was greater than zero (one-sample $t$ test, $p<0.0001 ; t=$ $6.50 ; n=29)$, i.e., B64 hyperpolarization extended the duration
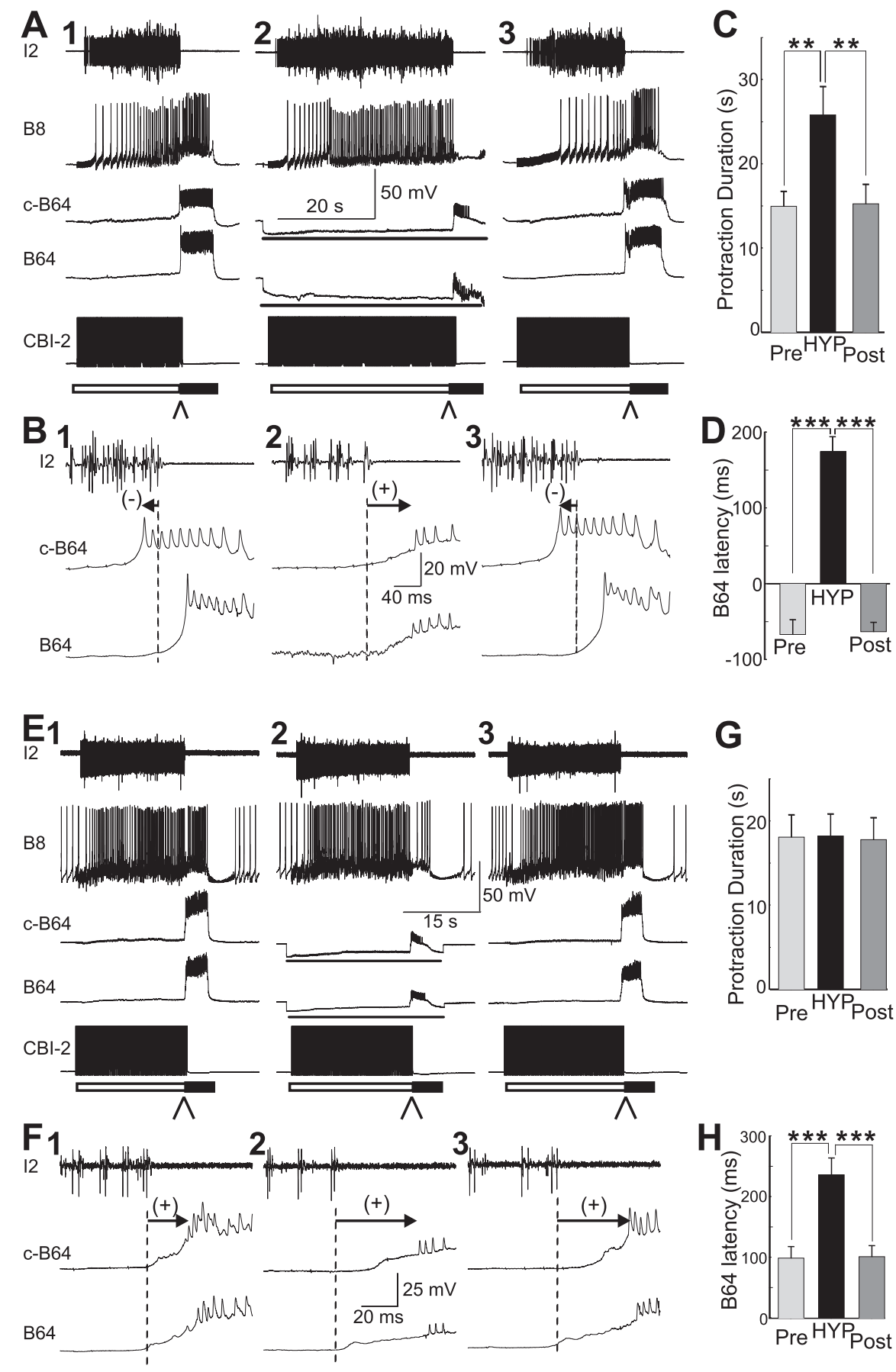

Figure 8. Effect of bilateral hyperpolarization of $\mathrm{B} 64$ on protraction duration and $\mathrm{B} 64$ latency in $\mathrm{CB}$-2-elicited intermediate programs with a negative $B 64$ latency $(\boldsymbol{A}-\boldsymbol{D})$ or a positive B64 latency $(\boldsymbol{E}-\boldsymbol{H}) . \boldsymbol{A}, \boldsymbol{E}$, Illustrative examples. $\boldsymbol{A} \mathbf{1}, \boldsymbol{E 1}$, A single cycle of a $(B I-2$-elicited intermediate motor program.A2,E2, Bilateral hyperpolarization of B64 with $10 \mathrm{nA}$ current (lines below the B64traces) prolonged protraction duration in a (BI-2-elicited intermediate motor program with negative B64 latency $(\boldsymbol{A} 2)$ but had no effect on protraction duration in a $\mathrm{CB}$-2-elicited intermediate motor program with positive B64 latency (E2).A3,E3,A third intermediate program without B64 hyperpolarization. The arrowheads below the bars point to the phase transition from protraction to retraction. $\boldsymbol{B} 1-\boldsymbol{B} \mathbf{3}, \boldsymbol{F 1}-\boldsymbol{F}$, Expanded view of the records from $\boldsymbol{A} \mathbf{1}-\boldsymbol{A} \mathbf{3}$ and $\boldsymbol{E} 1-\boldsymbol{E}$, respectively (at the places wherearrowheads are pointing to) shows B64latency. In $\boldsymbol{B}, \mathrm{B} 64$ latency was negative (B1), became positive when B64s werehyperpolarized (B2), and returned to negative without B64 hyperpolarization (B3). In $\boldsymbol{F}$, B64 latency was positive (F1), became more positive when B64s were hyperpolarized $(\boldsymbol{F 2})$, and remained positive without B64 hyperpolarization $(\boldsymbol{F 3}) . \mathbf{C}, \boldsymbol{D}, \mathbf{G}, \boldsymbol{H}$, Grouped data show protraction duration $(\boldsymbol{C}, \boldsymbol{G})$ and $B 64$ latency $(\boldsymbol{D}, \boldsymbol{H})$ when both B64s were hyperpolarized (HYP) compared with those in control conditions (Pre, before B64 hyperpolarization; Post, after B64hyperpolarization) in theintermediateprograms. Posthoctestswith Bonferroni'scorrections, ${ }^{* *} p<0.01$; ${ }^{* * *} p<0.001$. ErrorbarindicateSEM. 


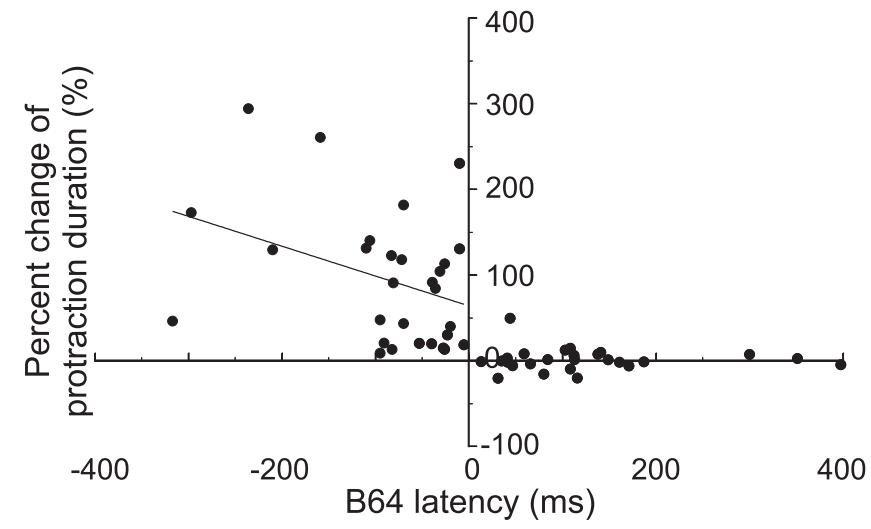

Figure 9. Plot of B64 latency versus percentage change of protraction duration for all B64 hyperpolarization experiments. Percentage change of protraction duration is defined as the difference between protraction duration during B64 hyperpolarization and that during controls divided by protraction duration during controls and then multiplied by 100 . A linear regression line is shown for data points when B64 latency is negative. The correlation coefficient is 0.13 . Linear regression is also performed for data points when $B 64$ latency is positive but is not shown because the slope of the line $(-0.00004)$ is close to zero, and the line would primarily overlap with the $x$-axis.

correlation coefficient $\left(r^{2}\right)$ when B64 latencies were positive was 0.0009 , and when B64 latencies were negative was 0.13 . The near zero correlation coefficient when B64 latencies were positive is expected because B64 hyperpolarization had no significant effect on protraction duration. Conversely, the weak correlation when B64 latencies were negative is consistent with the idea that the B64-independent means of protraction termination may make an important contribution to protraction termination when B64 is hyperpolarized, and these B64-independent actions are not highly correlated with how negative B64 latencies are. Also, if one considers that, under control conditions, B64 latencies are on the order of tens of milliseconds, whereas hyperpolarizations of B64 extend the duration of protractions by $>10 \mathrm{~s}$, it is perhaps not surprising that, given the biological variability in the feeding system of Aplysia (Horn et al., 2004; Lum et al., 2005; Ye et al., 2006; Nargeot et al., 2007), the correlation was weak.

\section{Dynamic change of B64 latency during program buildup and biasing}

The above data support the possibility that the latencies of B64 and the effects of B64 hyperpolarization are a reflection of the state of the network. If this is the case, it should be possible to experimentally change positive latencies into negative ones, and vice versa, by altering the state of the network. Previous work showed that previous history of network activity produces lasting changes in the state of the system (Proekt et al., 2004, 2007; Brezina et al., 2005; Zhurov et al., 2005). We used previously described experimental paradigms (Proekt et al., 2004, 2007) to manipulate the state of the network and characterized the resulting changes in B64 latency.

In the first group of experiments (buildup), we used preparations in which initially 120 -s-ISI CBI-2 stimulation generated intermediate motor programs that were characterized by positive B64 latencies. Then, 30-s-ISI CBI-2 stimulation was used until eight motor programs were elicited. Such a stimulation paradigm tends to make programs more ingestive. Figure $10 \mathrm{~A}$ shows that, as described previously (Proekt et al., 2004, 2007), this is indeed what happened because the first program of the eight programs was intermediate (Fig. 10A1), whereas the last program was ingestive (Fig. 10A2). The latency of B64 firing was positive in the
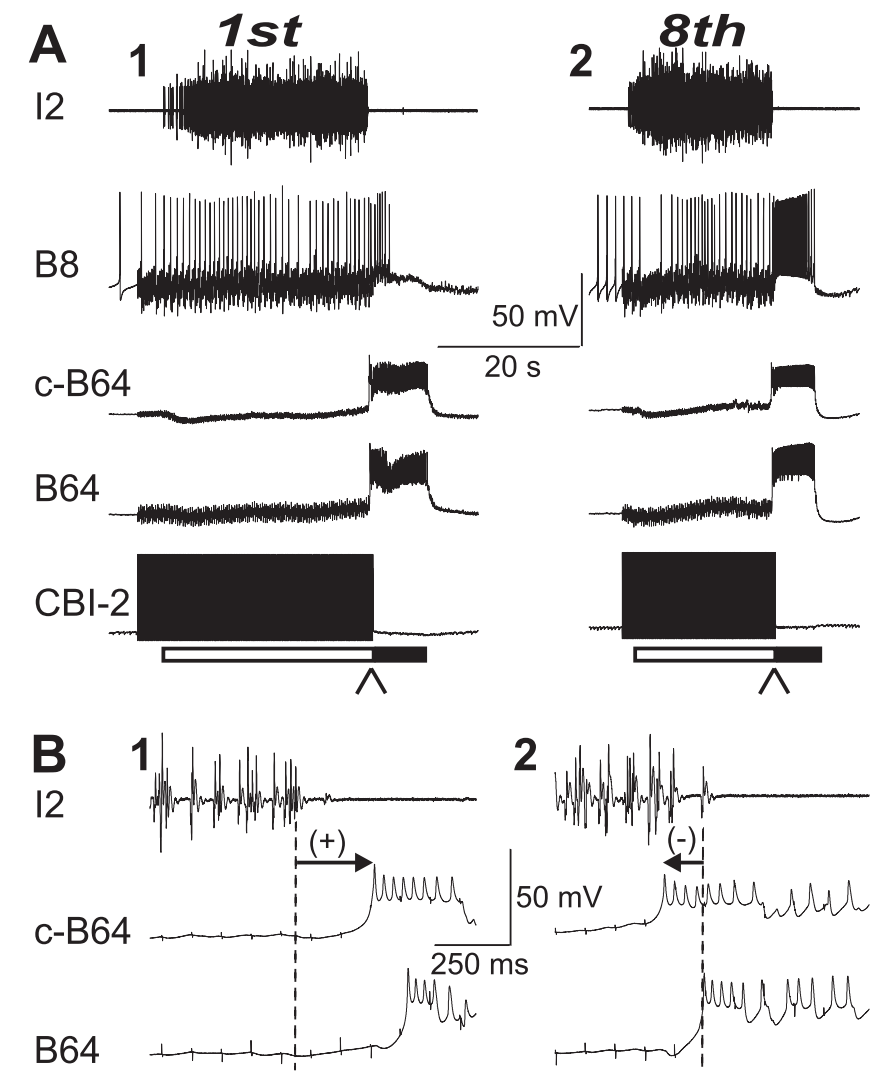

Figure 10. Dynamic change of B64 latency during buildup of motor programs elicited by CBI-2. CBI-2 was stimulated to induce a series of eight single-cycle motor programs using the 30-s-ISI stimulation paradigm (interstimulation interval of $30 \mathrm{~s}$ ), and motor programs became more ingestive as more programs were elicited. $\boldsymbol{A}$, Illustrative examples from a single preparation. $\boldsymbol{B} 1, \boldsymbol{B} 2$, Expanded view of the records from $\boldsymbol{A} \mathbf{1}$ and $\boldsymbol{A} \mathbf{2}$, respectively (at the places where arrowheads are pointing to) shows $B 64$ latency. $\boldsymbol{A} \mathbf{1}, \boldsymbol{B} 1$, The first motor program elicited by CBI-2 stimulation was intermediate (A1) and had a positive B64 latency (B1). A2, B2, The eighth motor program elicited by $\mathrm{CBI}-2$ stimulation became ingestive $(\boldsymbol{A 2})$ and now had a negative B64 latency (B2).

first program (Fig. 10B1) and became negative in the last program (Fig. 11 B2). Grouped data $(n=12)$ show that the mean B64 latency in the first cycle was positive $(95.82 \pm 27.57 \mathrm{~ms})$, whereas in the last cycle, the mean B64 latency was negative $(-95.00 \pm$ $33.3 \mathrm{~ms})$. This difference was statistically significant $(p<0.01$; $t=4.40$, paired $t$ test).

In the second series of experiments (biasing), we used a 30-sISI CBI-2 stimulation paradigm to establish reliable ingestive programs $(n=5)$. In these programs, B64 latency was negative and the programs were ingestive (Fig. $11 A 1, C 1$ ). We then suspended CBI-2 stimulation and replaced it with a 5 min EN stimulation that elicited egestive programs (data not shown). The stimulation was then switched back to CBI-2. As described previously (Proekt et al., 2004, 2007), the motor program elicited by the first CBI-2 stimulation immediately after EN stimulation was now egestive (Fig. $11 \mathrm{B1}$ ). We found that this was accompanied by the switch in B64 latency from negative (Fig. 11C1) to positive (Fig. 11D1). On average, after EN stimulation, the mean B64 latencies were $55.75 \pm 29.54 \mathrm{~ms}$, whereas they were $-55.25 \pm$ $11.07 \mathrm{~ms}$ before EN stimulation $(n=5)$ (Fig. $11 E 1)$. The difference between the before-EN and the after-EN stimulation was statistically significant ( $p<0.05 ; t=4.28$, paired $t$ test). In four of the five preparations in which we measured B64 latencies, we also examined the effects of B64 hyperpolarization on protrac- 
tion duration. Whereas before EN stimulation, B64 hyperpolarization prolonged the protraction duration of CBI-2-elicited ingestive motor programs (Fig. 11, compare $A 1, A 2)$, after EN stimulation, B64 hyperpolarization no longer affected the duration of protraction of the now egestive programs elicited by CBI-2 (Fig. 11, compare B1, B2). Analysis of grouped data of protraction duration before EN stimulation showed significant differences between the three groups ("Pre," "HYP," and "Post") $\left(F_{(2,6)}=7.66 ; p<0.05 ; n=4\right)$ (Fig. 11E2, left), whereas analysis of grouped data of protraction duration after EN stimulation showed no significant difference between the three groups $\left(F_{(2,6)}=\right.$ $0.23 ; p=0.80 ; n=4$ ) (Fig. $11 E 2$, right).

\section{Discussion}

Here, we demonstrate state-dependent changes in the functional role of interneuron B64 that maintains a similar pattern of sustained firing in different types of motor programs and is involved in phase transitions. This is in contrast to numerous studies in other systems in which network elements changed their function as a result of radical changes of the temporal phasing of their sustained activity. Because of such changes in phasing, neurons jumped between or fused networks that control distinct peripheral structures (Getting and Dekin, 1985; Hooper and Moulins, 1989; Dickinson et al., 1990; Meyrand et al., 1991; Weimann and Marder, 1994; Popescu and Frost, 2002; Jing and Gillette, 2003).

Even when the same peripheral structure is involved, a CPG element may change its function without a major change of the temporal characteristics of its sustained firing. Specifically, this happened when the network state changed attributable to alterations in contextual stimuli, e.g., the presence of peptides (Saideman et al., 2006; Thirumalai et al., 2006). These peptides changed the network speed without altering the type of motor program generated by the CPG. In contrast, here we show that alterations of the activity-dependent network states, which change the type of motor program, can also alter the function of B64 that implements phase transitions of motor programs. Network states induced by modification of contextual stimuli and network states that are activity dependent are fundamentally different in that the former states are directly controlled by the current stimulus conditions, whereas the latter states depend on the memory of the history of network activity and are expressed even when the stimulus conditions of the network remain constant. Most significantly, we showed that changes of activity-dependent network states critically modify
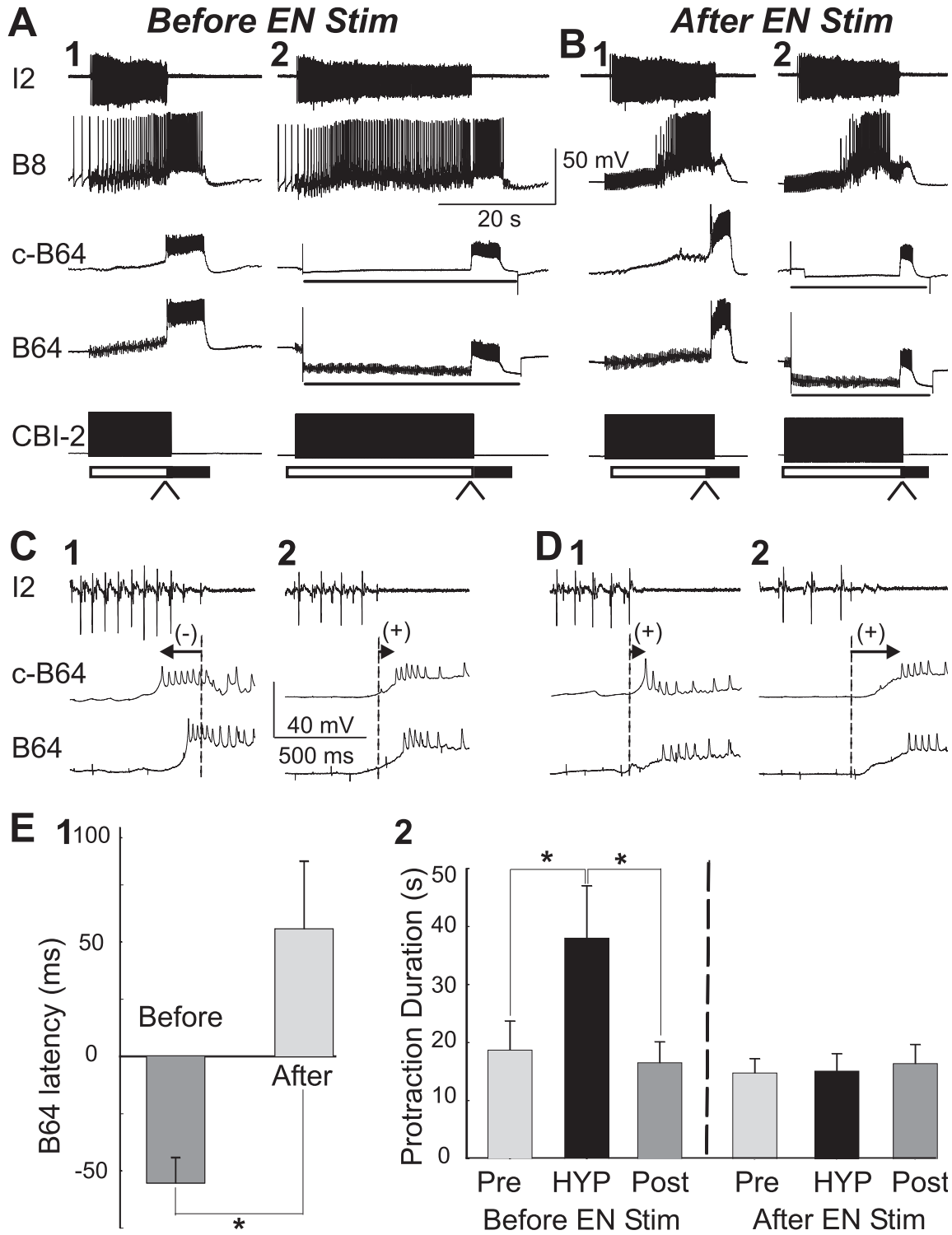

Figure 11. Dynamic change of $B 64$ latency during biasing of $C B I-2$-elicited motor programs by EN stimulation. $A, B$, Illustrative examples from a single preparation. $\mathbf{C 1}, \mathbf{C}, \mathbf{D 1}, \mathbf{D 2}$, Expanded view of the records from $\boldsymbol{A 1}, \boldsymbol{A} \mathbf{2}$ and $\mathbf{B 1}, \mathbf{B 2}$, respectively (at the places where arrowheads are pointing to), shows B64 latency. Eight cycles of motor programs were elicited by (BI-2 stimulation using the 30-s-ISI stimulation paradigm, and the eighth cycle, which is shown in $\boldsymbol{A 1}$ and $\mathbf{C 1}$, became ingestive (A1) and B64 latency was negative (C1). In four of five preparations, one of which is illustrated here, a ninth program was elicited while bilateral B64s were hyperpolarized to verify that protraction duration can be lengthened (A2) and B64 latency became positive (C2). Subsequently, CBI-2 stimulation was suspended and EN was continuously stimulated for 5 min to elicit 8-10 cycles of egestive motor programs (data not shown). Immediately after EN stimulation, CBI-2 stimulation (30-s-ISI stimulation paradigm) resumed, and the first cycle of the motor program, which is shown in $\mathbf{B 1}$ and $\mathbf{D 1}$, became egestive $(\boldsymbol{B 1})$ and $\mathrm{B} 64$ latency became positive (D1). Again, in four of five preparations, one of which is illustrated here, a second program was elicited while bilateral B64s were hyperpolarized to show that protraction duration did not change (B2) and B64 latency was more positive (D2). E1, Grouped data show that B64 latency was significantly different in $\mathrm{CBI}-2$-elicited motor programs obtained before EN stimulation (see $\mathrm{C1}$ ) versus that obtained after EN stimulation (see D1). ${ }^{*} p<0.05$. E2, Grouped data show that protraction duration in CBI-2-elicited motor programs can be significantly lengthened by bilateral B64 hyperpolarization before EN stimulation (compare $\boldsymbol{A} \mathbf{1}$ with $\boldsymbol{A}$ ), whereas B64 hyperpolarization no longer had an effect on protraction duration after EN stimulation (compare B1 with B2). Post hoc tests with Bonferroni's corrections, ${ }^{*} p<0.05$. Error bars indicate SEM. Pre, The first program induced by CBI-2; HYP, a program induced by $\mathrm{CBI}-2$ with hyperpolarization of both $\mathrm{B} 64$; ; Post, a recovery program induced by $\mathrm{CBI}-2$.

B64 spike-onset timing, thus leading to alterations in its function. In this context, it is worth noting that precise spike timing may be of critical importance for the expression of more complex forms of learning and memory (Robbe et al., 2006). A more complete picture of the role of spike timing in various forms of learning and memory will emerge as more examples are being studied in the future. 


\section{Phase transition and spike-onset timing}

Several observations indicate that B64 acts as a protraction terminator in some but not other types of motor programs. First, B64 hyperpolarization extended protraction duration in CBI-2elicited ingestive programs but not in other programs, e.g., ENelicited egestive programs. Second, B64 activation preceded protraction termination in CBI-2-elicited ingestive programs but followed protraction termination in EN-elicited egestive programs. Third, when B64 is hyperpolarized in ingestive programs, protraction is terminated before B64 begins to fire. Altogether, our data indicate that, within the feeding CPG, there exist at least two means of terminating protraction, B64-dependent and B64independent, and that these two means are related to differential timing of the onset of B64 spiking.

When a given phase-transition neuron displays a similar pattern of activity in two different motor programs, it may be assumed that this neuron also fulfills the same phase-terminating function in these different programs. Our present work showed this was not the case when two categorically distinct programs were induced. Furthermore, a shift of B64 spike-onset timing resulted in a change of its phase-terminating function. Potentially, these findings may also apply, although not yet proven, in vertebrate preparations based on previous work (Berkowitz and Stein, 1994; Pena et al., 2004). Conversely, in the crab, hyperpolarization of neuron LP is effective in changing program speed during application of one neuromodulator but not the other (Saideman et al., 2006; Thirumalai et al., 2006). Thus, it remains to be determined whether changes in the timing of the neurons that control phase transitions in these vertebrate and invertebrate preparations may be altered in a similar manner as that observed for B64.

Single vertebrate networks can generate programs that differ in speed, e.g., fast and slow walking, as well as programs that differ categorically, e.g., forward and backward locomotion (Jing and Weiss, 2005; Grillner, 2006). The invertebrate findings regarding the functional change of LP under different modulator influences affects program speed, and the functional role of B64 changes between different program types suggest that parallel principles of network functioning may operate in vertebrate networks, i.e., that different populations of neurons may be responsible for phase transitions when vertebrate CPGs generate different motor programs. Despite difficulties that one encounters in studying numerically large vertebrate networks, a recent genetic/molecular approach identified a spinal CPG neuron type that is involved in controlling the speed of locomotion (Gosgnach et al., 2006). It is therefore reasonable to expect that future vertebrate studies may uncover parallels with the invertebrate studies of the LP and B64 neurons.

The existence of multiple mechanisms that fulfill a similar function within a single CPG is not unique to the feeding CPG of Aplysia. Many CPGs are characterized by a highly distributed connectivity that often results in different neurons fulfilling a similar function. Consistent with this, in Aplysia, we found that protraction can also be terminated independently of B64, in addition to being terminated by B64. Specifically, B64 hyperpolarization experiments suggested that another neuron or neurons can terminate protraction albeit with a delay. Interestingly, such neurons may be synaptically connected to B64 because, in egestive programs in which protraction appears to be terminated in a B64-independent manner, B64 receives a strong excitatory input at the time of protraction termination. A similar excitatory input is observed in B64 when B64 is hyperpolarized and protraction is terminated by other neurons. It is thus possible that the same neurons that terminate protraction in egestive programs also terminate protraction in ingestive programs when B64 is hyperpolarized. At this time, however, we cannot exclude the possibility that more than two different neurons act as protraction terminators under different conditions. Given what is known about the feeding circuit of Aplysia, in principle one could construct several alternative models of how protraction is terminated under different conditions. However, such models would be highly speculative and therefore it is perhaps best to wait until more empirical data are available to construct them.

\section{State dependence}

Various CPGs generate different motor programs in response to the different triggering inputs (Chiel et al., 1988; Berkowitz and Stein, 1994; Church and Lloyd, 1994; Blitz et al., 2004; Jing et al., 2004, 2007). However, at different times, even when the same triggering stimulus is used, a network can generate different responses. This indicates that network-generated responses are also determined by the network state at the time when a motor program is generated. States can be established by the presence of specific contextual stimuli (Nolen and Hoy, 1984; Hedwig, 2000; Esch et al., 2002) or hormones/modulator presence (HarrisWarrick and Marder, 1991; Morgan et al., 2000, 2002; Jing and Weiss, 2001; Wu et al., 2003; Proekt et al., 2005; Tryba et al., 2006; Koh and Weiss, 2007). In addition to being affected by the context, the network state is also affected by the history of its recent activity (Nadim et al., 1999; Proekt and Weiss, 2003; Proekt et al., 2004, 2007).

In the Aplysia feeding CPG, the establishment of activitydependent network states is related to the history of activation of specific inputs. Repeated stimulation of CBI-2, which was shown previously to promote generation of ingestive programs in response to subsequent CBI-2 stimulation (Proekt et al., 2004, 2007), also promotes the protraction-terminating role of B64. In contrast, after repeated stimulation of the EN, which promotes generation of egestive programs in response to subsequent CBI-2 stimulation, B64 did not act as a protraction terminator (Fig. 11). Previous data showed that these different states were, respectively, associated with selective changes in the activity levels and synaptic outputs of interneurons B20 and B40 (Proekt et al., 2004,2007 ) that determine whether the radula closes during protraction or retraction and therefore determine the types of programs (Jing and Weiss, 2001, 2002). In contrast to B20 and B40, B64 affects the radula protraction/retraction movements, the two radula movements that maintain a fixed sequence in different programs. Thus, network states appear to affect not only the interneurons that promote expression of ingestive versus egestive programs but also those interneurons that are involved in the control of those aspects of programs that maintain a similar phasing in different programs. In conclusion, the control that network states exert in motor program generation may be more pervasive than previously assumed. Notably, the relatively simple feeding circuit of Aplysia allowed us to directly characterize how distinct network states influence spike timing, thereby altering neuronal functions.

Growing evidence suggests that vertebrate and invertebrate motor networks share a number of similarities. In vertebrate motor networks, phase transitions are often studied from the perspective of pacemakers, ionic mechanisms, or neuronal recruitment (Berkowitz and Stein, 1994; Pena et al., 2004; Grillner, 2006). To the best of our knowledge, the notion that the timing of the onset of neuronal firing may determine whether a neuron acts as a phase terminator has not yet been proposed. Thus, our find- 
ing on the role of timing mechanisms may add a novel perspective to the current thinking in the field of motor control.

\section{References}

Bartos M, Nusbaum MP (1997) Intercircuit control of motor pattern modulation by presynaptic inhibition. J Neurosci 17:2247-2256.

Berkowitz A, Stein PS (1994) Activity of descending propriospinal axons in the turtle hindlimb enlargement during two forms of fictive scratching: phase analyses. J Neurosci 14:5105-5119.

Blitz DM, Beenhakker MP, Nusbaum MP (2004) Different sensory systems share projection neurons but elicit distinct motor patterns. J Neurosci 24:11381-11390.

Brezina V, Horn CC, Weiss KR (2005) Modeling neuromuscular modulation in Aplysia. III. Interaction of central motor commands and peripheral modulatory state for optimal behavior. J Neurophysiol 93:1523-1556.

Chiel HJ, Kupfermann I, Weiss KR (1988) An identified histaminergic neuron can modulate the outputs of buccal-cerebral interneurons in Aplysia via presynaptic inhibition. J Neurosci 8:49-63.

Church PJ, Lloyd PE (1994) Activity of multiple identified motor neurons recorded intracellularly during evoked feedinglike motor programs in Aplysia. J Neurophysiol 72:1794-1809.

Combes D, Meyrand P, Simmers J (1999) Motor pattern specification by dual descending pathways to a lobster rhythm-generating network. J Neurosci 19:3610-3619.

Dembrow NC, Jing J, Brezina V, Weiss KR (2004) A specific synaptic pathway activates a conditional plateau potential underlying protraction phase in the Aplysia feeding central pattern generator. J Neurosci 24:5230-5238.

Dickinson PS, Mecsas C, Marder E (1990) Neuropeptide fusion of two motor-pattern generator circuits. Nature 344:155-158.

Esch T, Mesce KA, Kristan WB (2002) Evidence for sequential decision making in the medicinal leech. J Neurosci 22:11045-11054.

Evans CG, Jing J, Proekt A, Rosen SC, Cropper EC (2003) Frequencydependent regulation of afferent transmission in the feeding circuitry of Aplysia. J Neurophysiol 90:3967-3977.

Flamm RE, Harris-Warrick RM (1986) Aminergic modulation in lobster stomatogastric ganglion. I. Effects on motor pattern and activity of neurons within the pyloric circuit. J Neurophysiol 55:847-865.

Getting PA (1989) Emerging principles governing the operation of neural networks. Annu Rev Neurosci 12:185-204.

Getting PA, Dekin MS (1985) Mechanisms of pattern generation underlying swimming in Tritonia. IV. Gating of central pattern generator. J Neurophysiol 53:466-480.

Gosgnach S, Lanuza GM, Butt SJ, Saueressig H, Zhang Y, Velasquez T, Riethmacher D, Callaway EM, Kiehn O, Goulding M (2006) V1 spinal neurons regulate the speed of vertebrate locomotor outputs. Nature $440: 215-219$.

Grillner S (2006) Biological pattern generation: the cellular and computational logic of networks in motion. Neuron 52:751-766.

Harris-Warrick RM, Marder E (1991) Modulation of neural networks for behavior. Annu Rev Neurosci 14:39-57.

Hedwig B (2000) Control of cricket stridulation by a command neuron: efficacy depends on the behavioral state. J Neurophysiol 83:712-722.

Hooper SL, Moulins M (1989) Switching of a neuron from one network to another by sensory-induced changes in membrane properties. Science 244:1587-1589.

Horn CC, Zhurov Y, Orekhova IV, Proekt A, Kupfermann I, Weiss KR, Brezina V (2004) Cycle-to-cycle variability of neuromuscular activity in Aplysia feeding behavior. J Neurophysiol 92:157-180.

Hurwitz I, Susswein AJ (1996) B64, a newly identified central pattern generator element producing a phase switch from protraction to retraction in buccal motor programs of Aplysia californica. J Neurophysiol 75:1327-1344.

Hurwitz I, Neustadter D, Morton DW, Chiel HJ, Susswein AJ (1996) Activity patterns of the $\mathrm{B} 31 / \mathrm{B} 32$ pattern initiators innervating the $\mathrm{I} 2$ muscle of the buccal mass during normal feeding movements in Aplysia californica. J Neurophysiol 75:1309-1326.

Hurwitz I, Kupfermann I, Susswein AJ (1997) Different roles of neurons B63 and B34 that are active during the protraction phase of buccal motor programs in Aplysia californica. J Neurophysiol 78:1305-1319.

Hurwitz I, Cropper EC, Vilim FS, Alexeeva V, Susswein AJ, Kupfermann I,
Weiss KR (2000) Serotonergic and peptidergic modulation of the buccal mass protractor muscle (I2) in Aplysia. J Neurophysiol 84:2810-2820.

Hurwitz I, Kupfermann I, Weiss KR (2003) Fast synaptic connections from CBIs to pattern-generating neurons in Aplysia: initiation and modification of motor programs. J Neurophysiol 89:2120-2136.

Hurwitz I, Susswein AJ, Weiss KR (2005) Transforming tonic firing into a rhythmic output in the Aplysia feeding system: presynaptic inhibition of a command-like neuron by a CPG element. J Neurophysiol 93:829-842.

Jing J, Gillette R (2003) Directional avoidance turns encoded by single interneurons and sustained by multifunctional serotonergic cells. J Neurosci 23:3039-3051.

Jing J, Weiss KR (2001) Neural mechanisms of motor program switching in Aplysia. J Neurosci 21:7349-7362.

Jing J, Weiss KR (2002) Interneuronal basis of the generation of related but distinct motor programs in Aplysia: implications for current neuronal models of vertebrate intralimb coordination. J Neurosci 22:6228-6238.

Jing J, Weiss KR (2005) Generation of variants of a motor act in a modular and hierarchical motor network. Curr Biol 15:1712-1721.

Jing J, Vilim FS, Wu JS, Park JH, Weiss KR (2003) Concerted GABAergic actions of Aplysia feeding interneurons in motor program specification. J Neurosci 23:5283-5294.

Jing J, Cropper EC, Hurwitz I, Weiss KR (2004) The construction of movement with behavior-specific and behavior-independent modules. J Neurosci 24:6315-6325.

Jing J, Vilim FS, Horn CC, Alexeeva V, Hatcher NG, Sasaki K, Yashina I, Kupfermann I, Sweedler JV, Weiss KR (2007) From hunger to satiety: reconfiguration of a feeding network by Aplysia neuropeptide Y. J Neurosci 27:3490-3502.

Koh HY, Weiss KR (2007) Activity-dependent peptidergic modulation of the plateau-generating neuron B64 in the feeding network of Aplysia. J Neurophysiol 97:1862-1867.

Lum CS, Zhurov Y, Cropper EC, Weiss KR, Brezina V (2005) Variability of swallowing performance in intact, freely feeding Aplysia. J Neurophysiol 94:2427-2446.

Marder E, Calabrese RL (1996) Principles of rhythmic motor pattern generation. Physiol Rev 76:687-717.

Meyrand P, Simmers J, Moulins M (1991) Construction of a patterngenerating circuit with neurons of different networks. Nature 351:60-63.

Morgan PT, Perrins R, Lloyd PE, Weiss KR (2000) Intrinsic and extrinsic modulation of a single central pattern generating circuit. J Neurophysiol 84:1186-1193.

Morgan PT, Jing J, Vilim FS, Weiss KR (2002) Interneuronal and peptidergic control of motor pattern switching in Aplysia. J Neurophysiol 87:49-61.

Morton DW, Chiel HJ (1993) In vivo buccal nerve activity that distinguishes ingestion from rejection can be used to predict behavioral transitions in Aplysia. J Comp Physiol 172:17-32.

Nadim F, Manor Y, Kopell N, Marder E (1999) Synaptic depression creates a switch that controls the frequency of an oscillatory circuit. Proc Natl Acad Sci USA 96:8206-8211.

Nargeot R, Baxter DA, Byrne JH (1997) Contingent-dependent enhancement of rhythmic motor patterns: an in vitro analog of operant conditioning. J Neurosci 17:8093-8105.

Nargeot R, Petrissans C, Simmers J (2007) Behavioral and in vitro correlates of compulsive-like food seeking induced by operant conditioning in Aplysia. J Neurosci 27:8059-8070.

Nolen TG, Hoy RR (1984) Initiation of behavior by single neurons: the role of behavioral context. Science 226:992-994.

Nusbaum MP, Beenhakker MP (2002) A small-systems approach to motor pattern generation. Nature 417:343-350.

Nusbaum MP, Marder E (1989) A modulatory proctolin-containing neuron (MPN). II. State-dependent modulation of rhythmic motor activity. J Neurosci 9:1600-1607.

Pearson KG (1995) Proprioceptive regulation of locomotion. Curr Opin Neurobiol 5:786-791.

Pena F, Parkis MA, Tryba AK, Ramirez JM (2004) Differential contribution of pacemaker properties to the generation of respiratory rhythms during normoxia and hypoxia. Neuron 43:105-117.

Popescu IR, Frost WN (2002) Highly dissimilar behaviors mediated by a multifunctional network in the marine mollusk Tritonia diomedea. J Neurosci 22:1985-1993.

Proekt A, Weiss KR (2003) Convergent mechanisms mediate preparatory 
states and repetition priming in the feeding network of Aplysia. J Neurosci 23:4029-4033.

Proekt A, Brezina V, Weiss KR (2004) Dynamical basis of intentions and expectations in a simple neuronal network. Proc Natl Acad Sci USA 101:9447-9452.

Proekt A, Vilim FS, Alexeeva V, Brezina V, Friedman A, Jing J, Li L, Zhurov Y, Sweedler JV, Weiss KR (2005) Identification of a new neuropeptide precursor reveals a novel source of extrinsic modulation in the feeding system of Aplysia. J Neurosci 25:9637-9648.

Proekt A, Jing J, Weiss KR (2007) Multiple contributions of an inputrepresenting neuron to the dynamics of the Aplysia feeding network. J Neurophysiol 97:3046-3056.

Robbe D, Montgomery SM, Thome A, Rueda-Orozco PE, McNaughton BL, Buzsaki G (2006) Cannabinoids reveal importance of spike timing coordination in hippocampal function. Nat Neurosci 9:1526-1533.

Rosen SC, Teyke T, Miller MW, Weiss KR, Kupfermann I (1991) Identification and characterization of cerebral-to-buccal interneurons implicated in the control of motor programs associated with feeding in Aplysia. J Neurosci 11:3630-3655.

Rossignol S, Dubuc R, Gossard JP (2006) Dynamic sensorimotor interactions in locomotion. Physiol Rev 86:89-154.
Saideman SR, Christie AE, Torfs P, Huybrechts J, Schoofs L, Nusbaum MP (2006) Actions of kinin peptides in the stomatogastric ganglion of the crab Cancer borealis. J Exp Biol 209:3664-3676.

Thirumalai V, Prinz AA, Johnson CD, Marder E (2006) Red pigment concentrating hormone strongly enhances the strength of the feedback to the pyloric rhythm oscillator but has little effect on pyloric rhythm period. J Neurophysiol 95:1762-1770.

Tryba AK, Pena F, Ramirez JM (2006) Gasping activity in vitro: a rhythm dependent on 5-HT2A receptors. J Neurosci 26:2623-2634.

Weimann JM, Marder E (1994) Switching neurons are integral members of multiple oscillatory networks. Curr Biol 4:896-902.

Weiss KR, Chiel HJ, Kupfermann I (1986) Sensory function and gating of histaminergic neuron C2 in Aplysia. J Neurosci 6:2416-2426.

Wu JS, Jing J, Diaz-Rios M, Miller MW, Kupfermann I, Weiss KR (2003) Identification of a GABA-containing cerebral-buccal interneuron-11 in Aplysia californica. Neurosci Lett 341:5-8.

Ye H, Morton DW, Chiel HJ (2006) Neuromechanics of coordination during swallowing in Aplysia californica. J Neurosci 26:1470-1485.

Zhurov Y, Proekt A, Weiss KR, Brezina V (2005) Changes of internal state are expressed in coherent shifts of neuromuscular activity in Aplysia feeding behavior. J Neurosci 25:1268-1280. 